\title{
Laboratory rotational spectroscopy of acrylamide and a search for acrylamide and propionamide toward Sgr B2(N) with ALMA*
}

\author{
L. Kolesniková1 ${ }^{1}$, A. Belloche ${ }^{2} \oplus$, J. Koucký ${ }^{1}$, E. R. Alonso ${ }^{3}$, R. T. Garrod ${ }^{4} \oplus$, K. Luková1 ${ }^{1}$, \\ K. M. Menten ${ }^{2}$, H. S. P. Müller ${ }^{5} \odot$, P. Kania ${ }^{1}$, and Š. $\operatorname{Urban}^{1}$ \\ ${ }^{1}$ Department of Analytical Chemistry, University of Chemistry and Technology, Technická 5, 16628 Prague 6, Czech Republic \\ e-mail: lucie.kolesnikova@vscht.cz \\ 2 Max-Planck-Institut für Radioastronomie, Auf dem Hügel 69, 53121 Bonn, Germany \\ ${ }^{3}$ Grupo de Espectroscopia Molecular (GEM), Edificio Quifima, Área de Química-Física, Laboratorios de Espectroscopia y \\ Bioespectroscopia, Parque Científico UVa, Unidad Asociada CSIC, Universidad de Valladolid, 47011 Valladolid, Spain \\ ${ }^{4}$ Departments of Chemistry and Astronomy, University of Virginia, Charlottesville, VA 22904, USA \\ 5 Astrophysik/I. Physikalisches Institut, Universität zu Köln, Zülpicher Str. 77, 50937 Cologne, Germany
}

Received 14 October 2021 / Accepted 23 November 2021

\begin{abstract}
Context. Numerous complex organic molecules have been detected in the universe and among them are amides, which are considered as prime models for species containing a peptide linkage. In its backbone, acrylamide $\left(\mathrm{CH}_{2} \mathrm{CHC}(\mathrm{O}) \mathrm{NH}_{2}\right)$ bears not only the peptide bond, but also the vinyl functional group that is a common structural feature in many interstellar compounds. This makes acrylamide an interesting candidate for searches in the interstellar medium. In addition, a tentative detection of the related molecule propionamide $\left(\mathrm{C}_{2} \mathrm{H}_{5} \mathrm{C}(\mathrm{O}) \mathrm{NH}_{2}\right)$ has been recently claimed toward $\mathrm{Sgr} \mathrm{B} 2(\mathrm{~N})$.

Aims. The aim of this work is to extend the knowledge of the laboratory rotational spectrum of acrylamide to higher frequencies, which would make it possible to conduct a rigorous search for interstellar signatures of this amide using millimeter wave astronomy. Methods. We measured and analyzed the rotational spectrum of acrylamide between 75 and $480 \mathrm{GHz}$. We searched for emission of acrylamide in the imaging spectral line survey ReMoCA performed with the Atacama Large Millimeter/submillimeter Array toward Sgr B2(N). We also searched for propionamide in the same source. The astronomical spectra were analyzed under the assumption of local thermodynamic equilibrium.

Results. We report accurate laboratory measurements and analyses of thousands of rotational transitions in the ground state and two excited vibrational states of the most stable syn form of acrylamide. In addition, we report an extensive set of rotational transitions for the less stable skew conformer. Tunneling through a low energy barrier between two symmetrically equivalent configurations has been revealed for this higher-energy species. Neither acrylamide nor propionamide were detected toward the two main hot molecular cores of Sgr B2(N). We did not detect propionamide either toward a position located to the east of the main hot core, thereby undermining the recent claim of its interstellar detection toward this position. We find that acrylamide and propionamide are at least 26 and 14 times less abundant, respectively, than acetamide toward the main hot core Sgr B2(N1S), and at least 6 and 3 times less abundant, respectively, than acetamide toward the secondary hot core Sgr B2(N2).

Conclusions. A comparison with results of astrochemical kinetics model for related species suggests that acrylamide may be a few hundred times less abundant than acetamide, corresponding to a value that is at least an order of magnitude lower than the observational upper limits. Propionamide may be as little as only a factor of two less abundant than the upper limit derived toward Sgr B2(N1S). Lastly, the spectroscopic data presented in this work will aid future searches of acrylamide in space.
\end{abstract}

Key words. astrochemistry - ISM: molecules - line: identification - ISM: individual objects: Sagittarius B2 astronomical databases: miscellaneous

\section{Introduction}

The peptide bond, $-\mathrm{C}(=\mathrm{O}) \mathrm{NH}-$, that is found in amides is vital for the biological realm. It provides linkages between amino acids, giving rise to peptides, which are the key players in mediating both the molecular interactions underpinning present life on Earth and potentially the prebiotic processes that preceded it (Frenkel-Pinter et al. 2020; Weber \& Pizzarello 2006). Therefore, it is not surprising that the question of peptide bond formation represents a highly topical theme in different fields

\footnotetext{
${ }^{\star}$ Full Tables A.1. and A.4. are only available at the CDS via anonymous ftp to cdsarc.u-strasbg.fr (130.79.128.5) or via http: //cdsarc.u-strasbg.fr/viz-bin/cat/J/A+A/659/A111
}

and disciplines, including prebiotic astrochemistry (Ruiz-Mirazo et al. 2014; Sandford et al. 2020; Stolar et al. 2021).

In astronomical settings, amides and other peptide-like species are actively subjects of investigation in star-forming regions, solar-type protostars, and other environments (e.g., Halfen et al. 2011; Belloche et al. 2017; Mendoza et al. 2014; Ligterink et al. 2020, 2021; Gorai et al. 2020; Colzi et al. 2021). These studies uncover their formation in space and their role as nodes in chemical networks connecting other prebiotic molecules. Remarkable progress has been made in increasing the sensitivity of astronomical observing capabilities (Jørgensen et al. 2020; Tercero et al. 2021), opening up unique possibilities for observing new target compounds in lower abundances than before. A great deal of experimental work has concentrated 
on laboratory simulations designed to mimic the interstellar ices, providing compelling evidence that molecules containing the peptide bond can be formed abiotically (see, e.g., Kaiser et al. 2013; Ligterink et al. 2018; Frigge et al. 2018). Finally, the possible formation and destruction pathways of peptide-like molecules has become an important target in theoretical calculations and chemical modeling (see, e.g., Redondo et al. 2013, 2014; Rimola et al. 2018; Barone et al. 2015; Quénard et al. 2018). All these studies provide valuable information on the peptide bond chemistry in space from different viewpoints, but with a single goal in common: to contribute to deciphering of one of the most long-standing puzzles presented to humankind: the origins of life.

Laboratory rotational spectroscopy is one of the key pillars in decoding the prebiotic inventory of space. It has contributed to the detection of several amides, among other prebiotically interesting molecules (McGuire 2018) such as, for example, glycolaldehyde ( $\mathrm{CH}_{2} \mathrm{OHCHO}$; Hollis et al. 2000), aminoacetonitrile $\left(\mathrm{NH}_{2} \mathrm{CH}_{2} \mathrm{CN}\right.$; Belloche et al. 2008), hydroxylamine $\left(\mathrm{NH}_{2} \mathrm{OH}\right.$; Rivilla et al. 2020), and ethanolamine $\left(\mathrm{NH}_{2} \mathrm{CH}_{2} \mathrm{CH}_{2} \mathrm{OH}\right.$; Rivilla et al. 2021). The simplest member of the amide family, namely, formamide $\left(\mathrm{NH}_{2} \mathrm{CHO}\right)$, has been observed in spectral-line rich sources, that is: Sgr B2 (Rubin et al. 1971; Gottlieb et al. 1973; Nummelin et al. 1998; Turner 1989; Thiel et al. 2017), Orion KL (Turner 1989; Motiyenko et al. 2012), IRAS 16293-2422 (Kahane et al. 2013; Coutens et al. 2016) and, more recently, in a number of other sources (see, e.g., López-Sepulcre et al. 2019). Also, N-methylformamide $\left(\mathrm{CH}_{3} \mathrm{NHCHO}\right)$ was recently discovered toward the Sgr B2 and NGC 6334I star-forming regions (Belloche et al. 2017, 2019; Ligterink et al. 2020), in which its structural isomer acetamide $\left(\mathrm{CH}_{3} \mathrm{C}(\mathrm{O}) \mathrm{NH}_{2}\right)$ had also previously been detected (Hollis et al. 2006; Halfen et al. 2011; Belloche et al. 2013; Ligterink et al. 2020). Very recently, a tentative detection of propionamide $\left(\mathrm{CH}_{3} \mathrm{CH}_{2} \mathrm{C}(\mathrm{O}) \mathrm{NH}_{2}\right)$ toward $\mathrm{Sgr} \mathrm{B} 2$ was claimed by $\mathrm{Li}$ et al. (2021). These detections suggest that more peptide bond bearing molecules are yet to be detected.

Another species with the peptide link is acrylamide $\left(\mathrm{CH}_{2} \mathrm{CHC}(\mathrm{O}) \mathrm{NH}_{2}\right)$. It contains two hydrogen atoms fewer than propionamide does and it shares the vinyl functional group with other known interstellar compounds such as vinyl cyanide $\left(\mathrm{CH}_{2} \mathrm{CHCN}\right.$; Gardner \& Winnewisser 1975), vinyl alcohol $\left(\mathrm{CH}_{2} \mathrm{CHOH}\right.$; Turner \& Apponi 2001), propenal $\left(\mathrm{CH}_{2} \mathrm{CHCHO}\right.$; Hollis et al. 2004), propylene $\left(\mathrm{CH}_{2} \mathrm{CHCH}_{3}\right.$; Marcelino et al. 2007), vinylacetylene ( $\mathrm{CH}_{2} \mathrm{CHCCH}$; Cernicharo et al. 2021), and vinylcyanoacetylene $\left(\mathrm{CH}_{2} \mathrm{CHC}_{3} \mathrm{~N}\right.$; Lee et al. 2021).

Acrylamide is a complex organic molecule (COM) in the astronomical sense (i.e., containing six atoms or more; Herbst $\&$ van Dishoeck 2009). Since COMs might be present in multiple conformations which, in addition, might be governed with large amplitude motions and low-frequency vibrations, their dense rotational spectra might be difficult to interpret. Extensive spectroscopic studies of complex amides such as N-methylformamide (Belloche et al. 2017), glycolamide (SanzNovo et al. 2020), propiolamide (Alonso et al. 2021), and glycinamide (Kisiel et al. 2022) were thus conducted to enable their interstellar hunt in the millimeter wave region. However, this has not been the case so far for acrylamide, whose rotational spectrum has been studied only up to $60 \mathrm{GHz}$ (Marstokk et al. 2000). This makes predictions at higher frequencies rather uncertain impeding its rigorous search in space.

As an isolated molecule in the gas phase, acrylamide exists in the form of syn and skew conformers (see Fig. 1), the latter of which was found by Marstokk et al. (2000) to be 6.5(6) $\mathrm{kJ} \mathrm{mol}^{-1}$

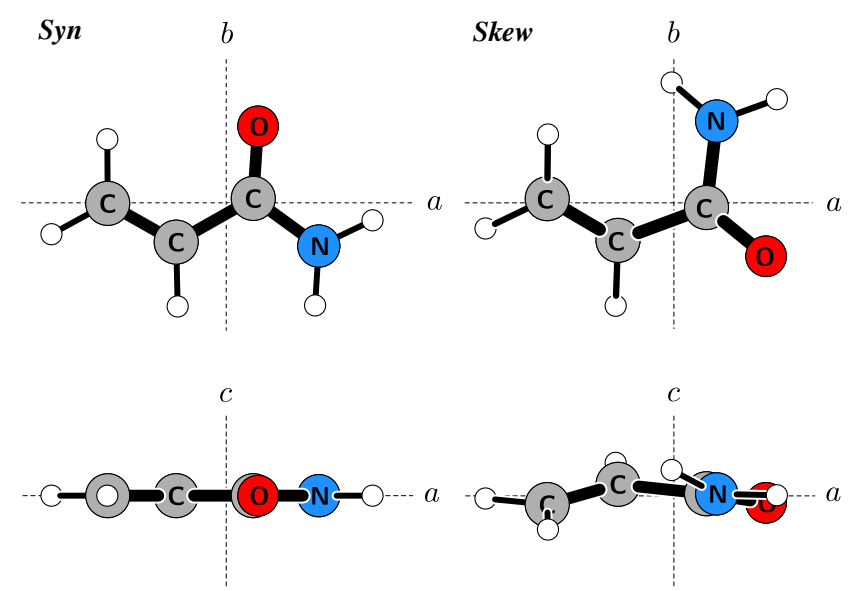

Fig. 1. Two conformers of acrylamide depicted in their principal $a b$ and $a c$ inertial planes.

or $543(50) \mathrm{cm}^{-1}$ less stable. In course of the present work, we measured the rotational spectrum of acrylamide between 75 and $480 \mathrm{GHz}$ and analyzed the spectral signatures of both species. The new measurements and analyses presented here provided precise laboratory information to search for this amide in the high-mass star-forming region Sgr B2(N), a possible source of large peptide molecules.

The remainder of the paper is structured as follows. Details about the laboratory spectroscopy are given in Sects. 2 and 3, while the search for acrylamide toward Sgr B2(N) is reported in Sect. 4. In the same section, we also report our nondetection of propionamide in Sgr B2(N) and thus, we cannot confirm its recently claimed interstellar detection. We discuss our results in Sect. 5 and provide our conclusions in Sect. 6.

\section{Experiments}

White crystalline acrylamide (m.p. $82-86{ }^{\circ} \mathrm{C}$ ) was obtained commercially and used without any further purification. The room-temperature rotational spectrum was measured in the frequency region $75-480 \mathrm{GHz}$ using two spectrometers. The lowest-frequency $(75-110 \mathrm{GHz})$ and the highest-frequency (170$480 \mathrm{GHz}$ ) sections were recorded using the millimeter wave spectrometer at the University of Valladolid (Daly et al. 2014), which is based on sequential multiplication of an Agilent synthesizer produced frequency by a set of active and passive multipliers from Virginia Diodes, Inc. The absorption free-space cell was a glass tube $(10 \mathrm{~cm}$ diameter, $360 \mathrm{~cm}$ long). The Prague millimeter wave spectrometer (Kania et al. 2006), which is based on the same principle, was used to measure the rotational spectrum between 128 and $170 \mathrm{GHz}$. Here, the free-space cell was $280 \mathrm{~cm}$ long with a diameter of $8 \mathrm{~cm}$. The optical path length was doubled by a roof-top mirror in both cases. The synthesizer output was frequency modulated in both spectrometers (modulation frequency of 10.2 and $28 \mathrm{kHz}$ ) and the detection system was completed by a demodulation procedure achieved by a lock-in amplifier that was tuned to twice the modulation frequency. All spectra were registered with the sample pressure between 10 and $20 \mu$ bar by upward and downward frequency scanning and averaged. The individual spectral sections were merged into a single spectrum and subjected to the analysis using the Assignment and Analysis of Broadband Spectra (AABS) package (Kisiel et al. 2005, 2012). 

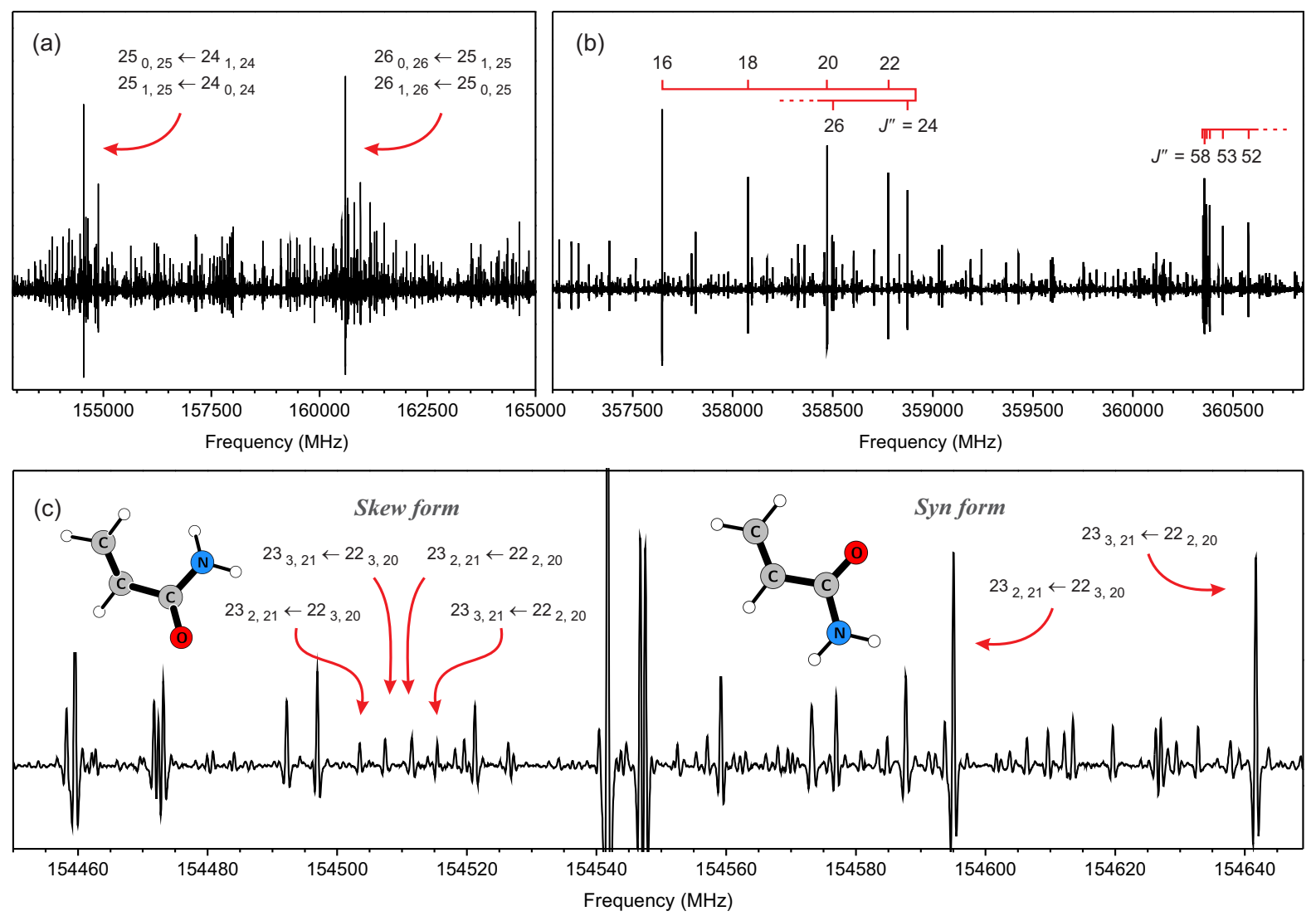

Fig. 2. Main features in the room-temperature rotational spectrum of acrylamide. (a) Strong lines of the syn conformer in the millimeter wave region consisting of a pair of $b$-type transitions involving $K_{a}=0,1$ and $K_{c}=J$ energy levels. $(b)$ Groups of $b$-type R-branch transitions near the sub-millimeter wave region. A diffuse group on the left embraces doubly degenerate transitions with the same $K_{a}$. Here, the leading transition is $J=K_{a}=17 \leftarrow 16$ and the value of $J$ increases by 2 while the value of $K_{a}$ decreases by 1 with each successive line. The compact group on the right encompasses degenerate transitions with two different $K_{a}$. In the present group, degenerate $K_{a}=0,1, K_{a}=1,2, \ldots K_{a}=6,7$ transitions are observed. Only $J$ quantum numbers are labeled with the red combs for simplicity. (c) A portion of the spectrum showing the rotational transitions of syn and skew acrylamide that involve $22_{2,20}$ and $22_{3,20}$ energy levels. While two $a$-type and two $b$-type transitions are observed for the skew conformer, only two $b$-type transitions are found for the syn form in agreement with their dipole moment components.

\section{Rotational spectra and analyses}

\subsection{Syn conformer}

The only available previous microwave study had shown that the syn conformer of acrylamide is essentially planar with a large dipole moment component along the $b$ principal inertial axis $\left(\left|\mu_{a}\right|=0.269(3) \mathrm{D},\left|\mu_{b}\right|=3.42(2) \mathrm{D},\left|\mu_{c}\right|=0.12(24) \mathrm{D}\right.$; Marstokk et al. 2000). At the initial stage of the analysis, predictions based on the spectroscopic constants from Marstokk et al. (2000) were used. Strong lines corresponding to pairs of $b$-type $R$-branch transitions $(J+1)_{1, J+1} \leftarrow J_{0, J}$ and $(J+1)_{0, J+1} \leftarrow J_{1, J}$ were readily assigned as they are visibly getting closer to each other with increasing $J$ quantum number. They finally coalesce into very prominent, doubly degenerate lines that stand out in the millimeter wave spectrum in Fig. 2a. On the other hand, groups of $b$-type $R$-branch transitions are gradually formed on the way to the sub-millimeter wave region (see Fig. 2b). Many $b$-type $Q$-branch transitions could also be identified throughout the spectrum. Finally, $a$-type transitions were searched for but could not be observed. Graphical Loomis-Wood-type plots produced with the AABS package (Kisiel et al. 2005, 2012) greatly facilitated the line assignments. Some of the observed lines were found to be partially split due to the nuclear quadrupole coupling interactions of a single ${ }^{14} \mathrm{~N}$ nucleus. They were not further taken into consideration due to the distorted line shapes.
We measured more than 2500 lines which encompass the rotational transitions with $J$ and $K_{a}$ quantum numbers up to 78 and 33, respectively. These transitions were ultimately merged with a selection of transitions from Marstokk et al. (2000) which were available in the microwave catalog from Ulm. Like our data set, this selection contained the rotational transitions for which the nuclear quadrupole hyperfine structure was expected to be collapsed. The fits and predictions were made in terms of Watson's $A$-reduced Hamiltonian in $\mathrm{I}^{\mathrm{r}}$-representation (Watson 1977). The broad coverage of transition types and quantum numbers ensured the precise determination of the rotational constants and full sets of the quartic and sextic centrifugal distortion constants. Their values are given in Table 1, while the list of measured transitions is provided in Table A.1.

Several excited vibrational states were observed for the syn conformer by Marstokk et al. (2000). The low-frequency C-C torsional mode in this conformer, which is labeled $v_{24}\left(A^{\prime \prime}\right)$ under the $C_{s}$ point group, was found to be a dominating contributor to the vibrational satellite pattern that was also observed in our records (see Fig. 3). Its frequency was estimated to $90(10) \mathrm{cm}^{-1}$ from the microwave relative intensity measurements (Marstokk et al. 2000). We were able to build upon results from Marstokk et al. (2000) and follow the successive excitation of this mode up to $v_{24}=4$. However, only $v_{24}=1$ and $v_{24}=2$ could be analyzed using the same Hamiltonian as for the ground state and their 
Table 1. Spectroscopic constants of syn acrylamide in the ground state (G.S.) and two excited vibrational states ( $A$-reduction, $\mathrm{I}^{\mathrm{r}}$-representation).

\begin{tabular}{|c|c|c|c|c|c|c|}
\hline & \multicolumn{3}{|c|}{ This work } & \multicolumn{3}{|c|}{ Marstokk et al. (2000) } \\
\hline & G.S. & $v_{24}=1$ & $v_{24}=2$ & G.S. & $v_{24}=1$ & $v_{24}=2$ \\
\hline$A / \mathrm{MHz}$ & $10732.819331(65)^{(a)}$ & $10660.711630(73)$ & $10594.14406(10)$ & $10732.8296(34)$ & $10660.7092(33)$ & $10594.1266(38)$ \\
\hline$B / \mathrm{MHz}$ & $4218.690256(41)$ & $4216.462772(50)$ & $4214.275015(87)$ & $4218.7012(13)$ & $4216.4693(11)$ & $4214.2765(14)$ \\
\hline$C / \mathrm{MHz}$ & 3030.752979 (33) & $3038.064954(36)$ & 3044.891587 (53) & $3030.7434(13)$ & $3038.0502(17)$ & $3044.8733(16)$ \\
\hline$\Delta_{J} / \mathrm{kHz}$ & $0.786816(13)$ & $0.804212(16)$ & $0.823406(30)$ & $0.7043(33)$ & $0.696(14)$ & $0.776(7)$ \\
\hline$\Delta_{J K} / \mathrm{kHz}$ & $3.755014(41)$ & $3.817382(52)$ & $3.91194(12)$ & $3.370(22)$ & $3.15(7)$ & $3.658(31)$ \\
\hline$\Delta_{K} / \mathrm{kHz}$ & $5.49540(11)$ & $5.17392(12)$ & $5.10038(19)$ & $5.403(8)$ & $5.100(24)$ & $5.055(15)$ \\
\hline$\delta_{J} / \mathrm{kHz}$ & $0.2227676(56)$ & $0.2240435(76)$ & $0.224728(17)$ & $0.2417(7)$ & $0.2394(26)$ & $0.2418(19)$ \\
\hline$\delta_{K} / \mathrm{kHz}$ & $3.361833(72)$ & $3.33546(10)$ & $3.36995(27)$ & $3.20(4)$ & $3.37(7)$ & $3.41(5)$ \\
\hline$\Phi_{J} / \mathrm{mHz}$ & $0.1763(16)$ & $0.2180(22)$ & $0.4452(56)$ & $-66(6)$ & $-208(33)$ & $-7(7)$ \\
\hline$\Phi_{J K} / \mathrm{mHz}$ & $1.361(16)$ & $-0.632(24)$ & $1.361^{(b)}$ & $-222(34)$ & $-2300(400)$ & $-650(110)$ \\
\hline$\Phi_{K J} / \mathrm{mHz}$ & $-30.836(56)$ & $-34.835(86)$ & $-47.69(10)$ & $-1540(130)$ & $-3500(500)$ & $-570(170)$ \\
\hline$\Phi_{K} / \mathrm{mHz}$ & $55.087(76)$ & $44.373(96)$ & $62.03(14)$ & $570(40)$ & $380(40)$ & $110(100)$ \\
\hline$\phi_{J} / \mathrm{mHz}$ & 0.09118 (77) & $0.0947(11)$ & $0.2032(30)$ & $1.70(34)$ & $-2.2(4)$ & $-10.7(26)$ \\
\hline$\phi_{J K} / \mathrm{mHz}$ & 1.104 (16) & $1.065(23)$ & $3.558(56)$ & $-600(50)$ & $-740(120)$ & $640(130)$ \\
\hline$\phi_{K} / \mathrm{mHz}$ & $40.43(11)$ & $17.00(18)$ & $31.43(27)$ & $1980(160)$ & $-1850(170)$ & $400(340)$ \\
\hline$J_{\min } / J_{\max }$ & $3 / 78$ & $4 / 78$ & $2 / 78$ & $1 / 75$ & $1 / 68$ & $2 / 64$ \\
\hline$K_{a}^{\min } / K_{a}^{\max }$ & $0 / 37$ & $0 / 38$ & $0 / 37$ & $0 / 37$ & $0 / 40$ & $0 / 39$ \\
\hline$N^{(c)}$ & 2710 & 2242 & 1599 & 354 & 322 & 317 \\
\hline$\sigma_{\mathrm{fit}}(d) / \mathrm{MHz}$ & 0.028 & 0.024 & 0.035 & 0.081 & 0.063 & 0.090 \\
\hline$\sigma_{\mathrm{w}}(e)$ & 0.93 & 0.98 & 0.98 & $\ldots$ & $\ldots$ & $\ldots$ \\
\hline
\end{tabular}

Notes. ${ }^{(a)}$ The numbers in parentheses are the parameter uncertainties in units of the last decimal digit. Their values are close to $1 \sigma$ standard uncertainties (67\% confidence level) because the unitless (weighted) deviation of the fit is close to 1.0. SPFIT/SPCAT program package (Pickett 1991) was used for the analysis. ${ }^{(b)}$ Fixed to the ground state value which is usually a preferred constraint against the zero or poorly determined value (Urban \& Sarka 1990; Koucký et al. 2013). ${ }^{(c)}$ Number of distinct frequency lines in the fit. ${ }^{(d)}$ Root mean square deviation of the fit. ${ }^{(e)}$ Unitless (weighted) deviation of the fit.

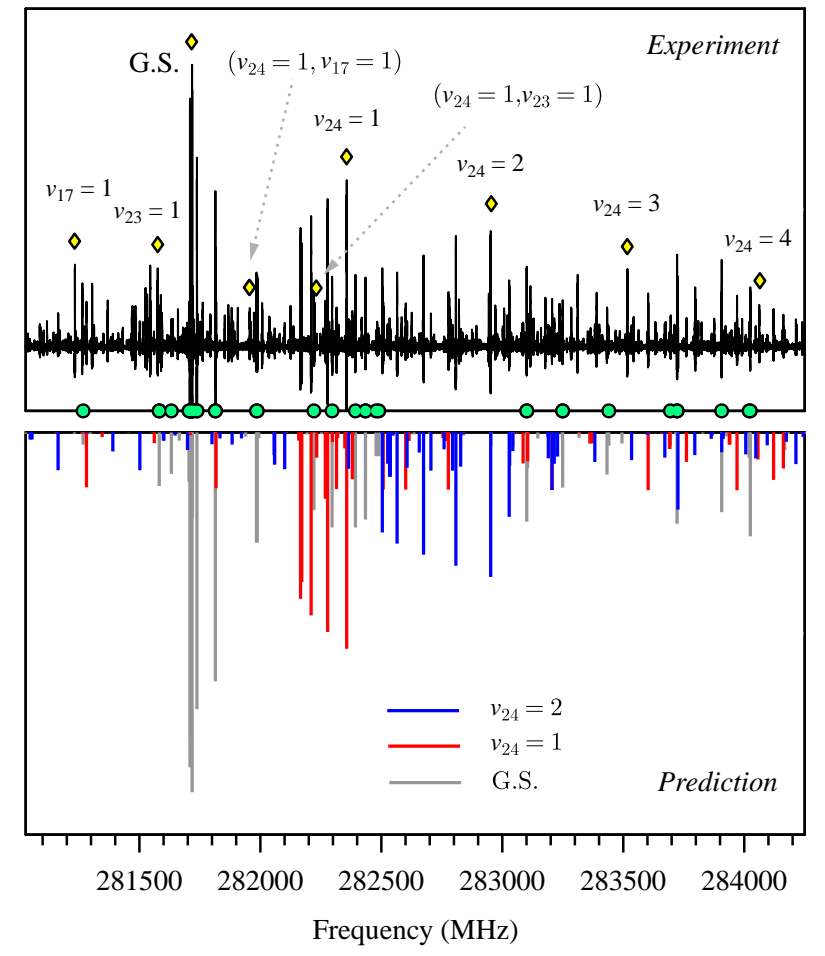

Fig. 3. Vibrational satellite pattern of the syn conformer in the millimeter wave spectrum of acrylamide. The upper panel shows a pair of degenerate $46_{1,46} \leftarrow 45_{0,45}$ and $46_{0,46} \leftarrow 45_{1,45}$ transitions for the ground state (G.S.) and the excited vibrational states (yellow diamonds). The green circle symbols represent the ground state lines included in the fit. The lower panel illustrates a stick reproduction of the ground state and $v_{24}=1,2$ spectra using the spectroscopic constants from Table 1 . spectroscopic constants are given in Table 1. The rotational transitions in $v_{24}=3$ and $v_{24}=4$ suffered from perturbations. These perturbations are not surprising since the manifold of vibrational energy levels become denser above $200 \mathrm{~cm}^{-1}$. In the neighborhood of $v_{24}=3$, which under the harmonic approximation can be estimated at $270 \mathrm{~cm}^{-1}$, there are first excited states of two other normal vibrational modes: $v_{23}\left(A^{\prime \prime}\right)$ and $v_{17}\left(A^{\prime}\right)$, which are associated with $\mathrm{NH}_{2}$ wagging and $\mathrm{C}=\mathrm{C}-\mathrm{C}$ in-plane bending motions, respectively. Their fundamentals were observed at $262.8 \mathrm{~cm}^{-1}$ and $307 \mathrm{~cm}^{-1}$ using infrared and Raman spectroscopies on gas phase (Kydd \& Dunham 1980) and solid phase samples (Duarte et al. 2005), respectively. Microwave relative intensity measurements provided values of $235(40) \mathrm{cm}^{-1}$ and $307(40) \mathrm{cm}^{-1}$ for $v_{23}=1$ and $v_{17}=1$, respectively (Marstokk et al. 2000). Due to the proximity of vibrational energy levels, significant vibration-rotation interactions might be thus manifested in pure rotational spectra of these states. This proximity is apparent from Fig. 3, where the same rotational transitions in $v_{24}=3, v_{23}=1$, and $v_{17}=1$ reveal very similar relative intensities. Practically the same situation seems to occur also for $v_{24}=4$, which is energetically accompanied by combination states $\left(v_{24}=1, v_{23}=1\right)$ and $\left(v_{24}=1, v_{17}=1\right)$.

We note that we were able to identify the rotational transitions in all these excited vibrational states in our records (see Fig. 3) in agreement with the assignments from Marstokk et al. (2000). Many lines for these states could be confidently assigned using the Loomis-Wood-type plots but could not be treated in the scope of the semi-rigid rotor Hamiltonian due to clear evidence of perturbations. An inspection of these perturbations suggests a rather complex and time-consuming treatment, probably requiring two three-state Hamiltonian fits. We are planning to discuss this quite intricate undertaking in a separate paper as 
Table 2. Partition functions and abundances for the two conformers of acrylamide.

\begin{tabular}{rrrrrrrrr}
\hline \hline & \multicolumn{3}{c}{ Syn conformer } & & \multicolumn{3}{c}{ Skew conformer } \\
\cline { 2 - 3 } \cline { 7 - 9 }$T(\mathrm{~K})$ & $Q_{\text {rot }}$ & $Q_{\text {vib }}$ & $(\%)^{(a)}$ & & $Q_{\text {rot }}{ }^{(b)}$ & $Q_{\text {vib }}$ & $(\%)$ \\
\hline 300.000 & 74866.65 & 8.11 & 89 & & 10980.80 & 6.65 & 11 \\
225.000 & 48631.40 & 3.97 & 95 & & 2962.15 & 3.37 & 5 \\
150.000 & 26465.75 & 2.08 & 99 & & 278.14 & 1.86 & 1 \\
75.000 & 9356.70 & 1.23 & 100 & & 0.51 & 1.18 & 0 \\
37.500 & 3309.64 & 1.03 & 100 & & 0.00 & 1.02 & 0 \\
18.750 & 1171.54 & 1.00 & 100 & & 0.00 & 1.00 & 0 \\
9.375 & 415.26 & 1.00 & 100 & & 0.00 & 1.00 & 0 \\
\hline
\end{tabular}

Notes. ${ }^{(a)}$ Conformer abundance calculated as $Q_{\mathrm{tot}}^{\text {syn }} /\left(Q_{\mathrm{tot}}^{\text {syn }}+Q_{\mathrm{tot}}^{\text {skew }}\right)$ where $Q_{\text {tot }}$ is the total partition function for a given conformer obtained as the product of $Q_{\text {rot }}$ and $Q_{\mathrm{vib}}{ }^{(b)}$ Accounts for the ground state tunneling doublet corrected for the energy of the skew conformer of $E_{\text {skew }}=543 \mathrm{~cm}^{-1}$ taken from Marstokk et al. (2000).

the outcome will have no bearing on the results reported in the present study.

Finally, we provide in Table 2 partition functions of syn acrylamide needed to estimate the column density. The rotational partition function $\left(Q_{\text {rot }}\right)$ was evaluated by summation of the Boltzmann factors over the energy levels in the ground vibrational state. We used the SPCAT program (Pickett 1991) to undertake this summation numerically employing the spectroscopic constants from Table 1 and all rotational states up to $J=120$. The vibrational partition function $\left(Q_{\text {vib }}\right)$ was obtained using the Eq. (3.60) of Gordy \& Cook (1970) by taking into account the frequencies of twenty-four normal vibrational modes from Table A.2.

\subsection{Skew conformer}

Spectroscopic constants from Marstokk et al. (2000) were used to search for rotational transitions of the less stable skew conformer in our records. Since the candidate lines were significantly weaker and were displaced by a few $\mathrm{MHz}$ from the predicted positions, a double check for the correct assignment in the very dense spectrum was the simultaneous observation of $a$-type and $b$-type transitions. Unlike the syn form, which possesses a sizable dipole moment component only along the $b$ principal axis, the skew conformer is endowed with large dipole moment components along the $a$ and $b$ axes $\left(\left|\mu_{a}\right|=2.81 \mathrm{D},\left|\mu_{b}\right|=\right.$ 3.02 D, $\left|\mu_{c}\right|=1.00 \mathrm{D}$ as calculated by Marstokk et al. 2000 at the MP2 $=$ full/cc-pVTZ level of theory). For certain values of $J$ this leads to the observations of easily discernible quartets in which the two $b$-type transitions straddle the pair of $a$-type transitions. This is clearly illustrated in Fig. 2c, which shows a small part of the spectrum at $154 \mathrm{GHz}$.

In this study, we assigned transitions belonging to the ground state and a higher-frequency satellite matching with what was assumed to be the first excited $\mathrm{C}-\mathrm{C}$ torsional state in Marstokk et al. (2000). Relative intensity measurements undertaken by Marstokk et al. (2000) placed this state into the energy window between 71 and $111 \mathrm{~cm}^{-1}$ above the ground state. On the other hand, our records indicate that this state lies significantly lower; its rotational lines apparently presented intensities very similar to those of the corresponding lines in the ground state. An examination of the Loomis-Wood-type plots made it further possible to identify several level-crossing perturbations. These

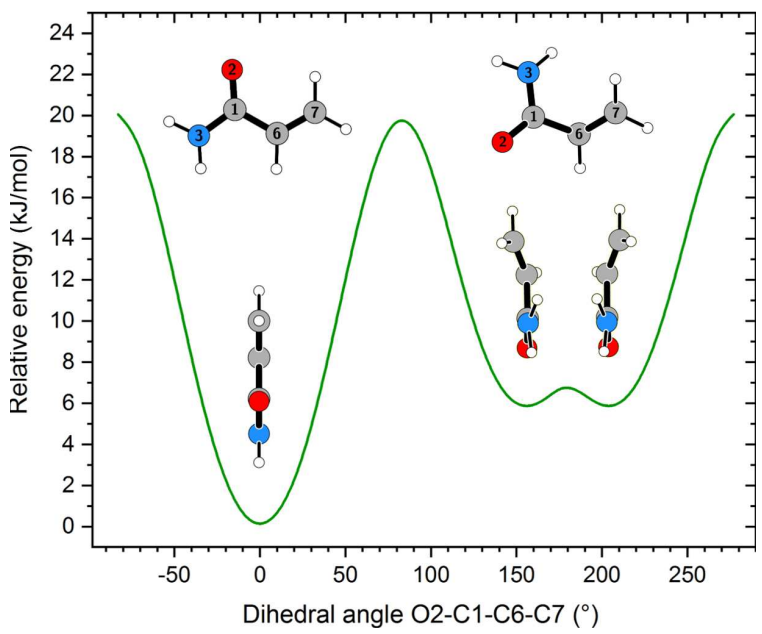

Fig. 4. Potential energy surface scan for the skeletal torsion calculated at the B3LYP/cc-pVTZ level of theory using the scan option of Gaussian 16 (Frisch et al. 2016). The energies were calculated in steps of $10^{\circ}$ of the dihedral angle O2-C1-C6-C7 while the other structural parameters were optimized under the verytight convergence criterion. The global minimum belongs to the planar syn conformer while for the higherenergy conformer two minima can be identified on the surface which correspond to two equivalent skew frameworks connected with a barrier of around $1 \mathrm{~kJ} \mathrm{~mol}^{-1}\left(84 \mathrm{~cm}^{-1}\right)$.

were observable in mirror-image form for the ground state and the satellite lines, presenting evidence that these two states are in close proximity and are in mutual interaction.

An explanation for the observation of such an interacting pair of states could be a double-minimum potential for skew acrylamide, which we subsequently confirmed by means of a potential energy surface scan for the rotation about the central $\mathrm{C}-\mathrm{C}$ bond. As shown in Fig. 4, two minima corresponding to two symmetrically equivalent non-planar skew structures are separated by a small barrier. In this scenario, the ground vibrational state, normally labeled $v=0$, is expected to be observed in the form of a doublet, the members of which are designated $0^{+}$ and $0^{-}$. These two states are often affected by vibration-rotation interactions as shown, for example, for glycinamide (Kisiel et al. 2022), cyanamide (Kisiel et al. 2013), 2-hydroxyacetonitrile (Margulès et al. 2017), and $a G g^{\prime}$ ethylene glycol (Christen \& Müller 2003). The identification of characteristic mirror-image perturbation spikes for various $K_{a}$, i.e. frequency shifts with respect to the unperturbed positions (see, e.g., Kisiel et al. 2013), allowed us to unambiguously assign the higher-frequency satellite to the higher-energy $0^{-}$state. Furthermore, their precise location in $J$ and $K_{a}$ allowed rather confident estimation of the energy difference $\Delta E=E^{\left(0^{-}\right)}-E^{\left(0^{+}\right)} \approx 13.8 \mathrm{~cm}^{-1}$. This value was used as an estimate in the fitting procedure that employed a two-state Hamiltonian in the following matrix form:

$H_{\mathrm{eff}}=\left(\begin{array}{cc}H_{\mathrm{rot}}^{\left(0^{+}\right)} & H_{\mathrm{cor}}^{\left(0^{+}, 0^{-}\right)} \\ H_{\mathrm{cor}}^{\left(0^{+}, 0^{-}\right)} & H_{\mathrm{rot}}^{\left(0^{+}\right)}+\Delta E\end{array}\right)=\left(\begin{array}{cc}H_{\mathrm{rot}}^{\left(0^{+}\right)} & H_{\mathrm{cor}}^{\left(0^{+}, 0^{-}\right)} \\ H_{\mathrm{cor}}^{\left(0^{+}, 0^{-}\right)} & H_{\mathrm{rot}}^{\left(0^{-}\right)}\end{array}\right)$,

where $H_{\mathrm{rot}}^{\left(0^{+}\right)}$and $H_{\mathrm{rot}}^{\left(0^{-}\right)}$represent Watson's $A$-reduced semi-rigid rotor Hamiltonians in $\mathrm{I}^{\mathrm{r}}$-representations (Watson 1977) for $0^{+}$ and $0^{-}$states, respectively. The off-diagonal coupling term $H_{\text {cor }}^{\left(0^{+}, 0^{-}\right)}$is based on a reduced axis system (RAS) approach of Pickett (1972) which was used to treat perturbations in molecular systems with symmetric double-minimum potentials such as cyanamide (Kisiel et al. 2013), propanal (Zingsheim et al. 2017), and methoxymethanol (Motiyenko et al. 2018) and takes 
Table 3. Spectroscopic constants for skew acrylamide in the ground state tunneling doublet (A-reduction, $\mathrm{I}^{\mathrm{r}}$-representation).

\begin{tabular}{|c|c|c|c|c|}
\hline & \multicolumn{2}{|c|}{ This work } & \multicolumn{2}{|c|}{ Marstokk et al. (2000) } \\
\hline & $0^{+}$ & $0^{-}$ & $0^{+}$ & $0^{-}$ \\
\hline$A / \mathrm{MHz}$ & $10049.46072(34)^{(b)}$ & 9996.61018 (39) & $10049.549(22)$ & $10002.8(5)$ \\
\hline$B / \mathrm{MHz}$ & $4287.87861(14)$ & 4291.77177 (19) & $4287.924(12)$ & $4292.160(24)$ \\
\hline$C / \mathrm{MHz}$ & 3035.794577 (67) & 3050.747935 (98) & $3035.806(12)$ & $3050.385(33)$ \\
\hline$\Delta_{J} / \mathrm{kHz}$ & $0.990767(85)$ & $0.95662(11)$ & $1.14(9)$ & $1.15(16)$ \\
\hline$\Delta_{J K} / \mathrm{kHz}$ & $3.47106(38)$ & $4.24560(48)$ & $4.20(12)$ & $3.35(18)$ \\
\hline$\Delta_{K} / \mathrm{kHz}$ & $7.0201(13)$ & $5.0704(15)$ & $8.0(6)$ & 8.0 \\
\hline$\delta_{J} / \mathrm{kHz}$ & $0.166200(44)$ & $0.194935(60)$ & $0.283(9)$ & 0.28 \\
\hline$\delta_{K} / \mathrm{kHz}$ & $5.03612(65)$ & $4.64196(99)$ & $3.23(15)$ & 3.2 \\
\hline$\Phi_{J} / \mathrm{Hz}$ & $0.002027(27)$ & $0.000986(39)$ & $\ldots$ & $\ldots$ \\
\hline$\Phi_{J K} / \mathrm{Hz}$ & $-0.00838(29)$ & $-0.00530(29)$ & $\ldots$ & $\ldots$ \\
\hline$\Phi_{K J} / \mathrm{Hz}$ & $-0.2776(11)$ & $-0.1182(12)$ & $\ldots$ & $\ldots$ \\
\hline$\Phi_{K} / \mathrm{Hz}$ & $0.1495(16)$ & $0.0781(18)$ & $\ldots$ & $\ldots$ \\
\hline$\phi_{J} / \mathrm{Hz}$ & $-0.000622(14)$ & $-0.000261(19)$ & $\ldots$ & $\ldots$ \\
\hline$\phi_{J K} / \mathrm{Hz}$ & $0.02621(24)$ & $0.00500(34)$ & $\ldots$ & $\ldots$ \\
\hline$\phi_{K} / \mathrm{Hz}$ & $-0.1939(23)$ & $-0.0737(26)$ & $\ldots$ & $\ldots$ \\
\hline$\Delta E / \mathrm{MHz}$ & 415050.2 & $4(55)$ & $\ldots$ & \\
\hline$\Delta E / \mathrm{cm}^{-1}$ & 13.84458 & $9(18)$ & $\ldots$ & \\
\hline$F_{b c} / \mathrm{MHz}$ & 4.08214 & (95) & $\ldots$ & \\
\hline$F_{b c}^{J} / \mathrm{kHz}$ & -0.1446 & $(66)$ & $\ldots$ & \\
\hline$F_{b c}^{K c} / \mathrm{kHz}$ & 1.1873 & 37) & $\cdots$ & \\
\hline$F_{b c}^{J J} / \mathrm{Hz}$ & 0.00156 & (53) & $\ldots$ & \\
\hline$F_{b c}^{b C} / \mathrm{Hz}$ & 0.0624 & 25) & $\ldots$ & \\
\hline$F_{a c}^{o c} / \mathrm{MHz}$ & -1.2209 & (94) & $\ldots$ & \\
\hline$F_{a c}^{J} / \mathrm{kHz}$ & -0.2239 & (41) & ... & \\
\hline$F_{a c}^{a c} / \mathrm{kHz}$ & 3.407 & & 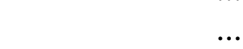 & \\
\hline$J_{\min } / J_{\max }$ & $4 / 76$ & $6 / 77$ & $4 / 27$ & $6 / 8$ \\
\hline$K_{a}^{\min } / K_{a}^{\max }$ & $0 / 24$ & $0 / 24$ & $0 / 8$ & $2 / 7$ \\
\hline$N^{(c)}$ & 1419 & 1255 & 53 & 14 \\
\hline$\sigma_{\text {fit }}(d) / \mathrm{MHz}$ & 0.040 & 0.035 & 0.196 & 0.129 \\
\hline$\sigma_{\mathrm{w}}(e)$ & 1.03 & 1.08 & $\ldots$ & $\ldots$ \\
\hline
\end{tabular}

Notes. ${ }^{(a)}$ The ground state and the excited C-C torsional state in Marstokk et al. (2000) correspond to $0^{+}$and $0^{-}$states, respectively. ${ }^{(b)}$ The numbers in parentheses are the parameter uncertainties in units of the last decimal digit. Their values are close to $1 \sigma$ standard uncertainties $(67 \%$ confidence level) because the unitless (weighted) deviation of the fit is close to 1.0. SPFIT/SPCAT program package (Pickett 1991) was used for the analysis. ${ }^{(c)}$ Number of distinct frequency lines in the fit. ${ }^{(d)}$ Root mean square deviation of the fit. ${ }^{(e)}$ Unitless (weighted) deviation of the fit.

the form:

$$
\begin{aligned}
H_{\mathrm{cor}}^{\left(0^{+}, 0^{-}\right)}= & \left(F_{b c}+F_{b c}^{J} J^{2}+F_{b c}^{K} J_{z}^{2}+\ldots\right)\left[J_{b}, J_{c}\right]_{+} \\
& \left(F_{a c}+F_{a c}^{J} J^{2}+F_{a c}^{K} J_{z}^{2}+\ldots\right)\left[J_{a}, J_{c}\right]_{+},
\end{aligned}
$$

where $F_{b c}$ and $F_{a c}$ are the interaction parameters. Their centrifugal distortion expansion terms $\left(F_{b c}^{J}, F_{b c}^{K}, \ldots\right)$ were systematically explored during the fitting procedure. A rather satisfactory fit, based on more than 2600 lines (our lines and those from Marstokk et al. 2000), was eventually reached with the choice for the parameter set given in Table 3. The list of measured rotational transitions is provided in Table A.4. Since the perturbations affect not only line frequencies but also line intensities (Christen \& Müller 2003; Kisiel et al. 2022), we tested the relative signs for the $F$ parameters with respect to those of the dipole moment components. The sign combination in Table 3 with positive $\mu_{a}$ and $\mu_{b}$ values reproduce the intensity alternations of perturbed transitions in the spectrum. We note that the same result is obtained if the signs of all $F$ parameters are reversed or those of all dipole moment components.

Final remarks concern the quartic and sextic centrifugal distortion constants in Table 3. Their values in $0^{+}$and $0^{-}$tunneling states are generally close to each other, however, a difference might be perceptible: for example, for $\Delta_{K}$. The main difficulty here was the high spectral density caused by the existence of the more stable syn conformer and its rotational lines in many excited vibrational states. It was sometimes rather difficult to evaluate whether the lines are obscured by these features or are still perturbed or simply are not observed due to their weakness and our limited signal to noise ratio. It is therefore possible that the perturbation contributions are treated incompletely. Nevertheless, the measured data set for skew acrylamide is relatively large and is reproduced near the experimental uncertainty using the spectroscopic parameters from Table 3.

\section{Search for acrylamide and related molecules toward Sgr B2(N)}

\subsection{Observations}

We used the imaging spectral line survey named Reexploring Molecular Complexity with ALMA (ReMoCA) performed with the Atacama Large Millimeter/submillimeter Array (ALMA) to search for acrylamide toward Sgr B2(N). The observational 
L. Kolesniková et al.: The rotational spectrum and ISM search of acrylamide

Table 4. Parameters of our best-fit LTE model of acetamide toward Sgr B2(N1S), and upper limits for propiolamide, acrylamide, and propionamide.

\begin{tabular}{|c|c|c|c|c|c|c|c|c|c|c|}
\hline & Molecule & Status $^{(a)}$ & $N_{\text {det }}(b)$ & $\begin{array}{c}\theta_{\mathrm{s}}{ }^{(c)} \\
\left({ }^{\prime \prime}\right)\end{array}$ & $\begin{array}{c}T_{\text {rot }}(d) \\
(\mathrm{K})\end{array}$ & $\begin{array}{c}N^{(e)} \\
\left(\mathrm{cm}^{-2}\right)\end{array}$ & $F_{\text {vib }}(f)$ & $\begin{array}{c}\Delta V^{(g)} \\
\left(\mathrm{km} \mathrm{s}^{-1}\right)\end{array}$ & $\begin{array}{c}V_{\text {off }}{ }^{(h)} \\
\left(\mathrm{km} \mathrm{s}^{-1}\right)\end{array}$ & $\frac{N_{\text {ref }}}{N}(i)$ \\
\hline Acetamide & $\mathrm{CH}_{3} \mathrm{C}(\mathrm{O}) \mathrm{NH}_{2}{ }^{(j) \star}$ & $\mathrm{d}$ & 153 & 2.0 & 160 & $4.1(17)$ & 1.16 & 5.0 & 0.0 & 1 \\
\hline Propiolamide & $\mathrm{HCCC}(\mathrm{O}) \mathrm{NH}_{2}{ }^{(k)}$ & $\mathrm{n}$ & 0 & 2.0 & 160 & $<8.5(15)$ & 1.18 & 5.0 & 0.0 & $>48$ \\
\hline Acrylamide & syn- $\mathrm{C}_{2} \mathrm{H}_{3} \mathrm{C}(\mathrm{O}) \mathrm{NH}_{2}$ & $\mathrm{n}$ & 0 & 2.0 & 160 & $<1.6(16)$ & 2.26 & 5.0 & 0.0 & $>26$ \\
\hline Propionamide & $\mathrm{C}_{2} \mathrm{H}_{5} \mathrm{C}(\mathrm{O}) \mathrm{NH}_{2}$ & $\mathrm{n}$ & 0 & 2.0 & 160 & $<2.9(16)$ & 4.16 & 5.0 & 0.0 & $>14$ \\
\hline
\end{tabular}

Notes. ${ }^{(a)}$ d: detection, n: nondetection. ${ }^{(b)}$ Number of detected lines (conservative estimate, see Sect. 3 of Belloche et al. 2016). One line of a given species may mean a group of transitions of that species that are blended together. ${ }^{(c)}$ Source diameter (FWHM). ${ }^{(d)}$ Rotational temperature. ${ }^{(e)}$ Total column density of the molecule. $x(y)$ means $x \times 10^{y} .{ }^{(f)}$ Correction factor that was applied to the column density to account for the contribution of vibrationally excited states, in the cases where this contribution was not included in the partition function of the spectroscopic predictions. ${ }^{(g)}$ Linewidth (FWHM). ${ }^{(h)}$ Velocity offset with respect to the assumed systemic velocity of Sgr B2(N1S), $V_{\text {sys }}=62 \mathrm{~km} \mathrm{~s}^{-1}$. ${ }^{(i)}$ Column density ratio, with $N_{\text {ref }}$ the column density of the previous reference species marked with a $\star .{ }^{(j)}$ The parameters were derived from the ReMoCA survey by Belloche et al. (2019). ${ }^{(k)}$ The upper limit was derived from the ReMoCA survey by Alonso et al. (2021).

strategy and the method employed to reduce the data of this survey were described in Belloche et al. (2019). We summarize here the main features. The survey extends from 84.1 to $114.4 \mathrm{GHz}$, with a spectral resolution of $488 \mathrm{kHz}\left(1.7\right.$ to $\left.1.3 \mathrm{~km} \mathrm{~s}^{-1}\right)$. It achieved a median angular resolution (HPBW) of $0.6^{\prime \prime}$, with values varying between $\sim 0.3^{\prime \prime}$ and $\sim 0.8^{\prime \prime}$. The median resolution corresponds to $\sim 4900$ au at the distance of Sgr B2 $(8.2 \mathrm{kpc}$, Reid et al. 2019). The interferometric observations were centered on the equatorial position $(\alpha, \delta)_{\mathrm{J} 2000}=\left(17^{\mathrm{h}} 47^{\mathrm{m}} 19^{\mathrm{s}} .87,-28^{\circ} 22^{\prime} 16^{\prime \prime} .0\right)$ that is located halfway between Sgr B2(N1) and Sgr B2(N2), the two main hot molecular cores of $\mathrm{Sgr}$ B2(N). These hot cores are separated by $4.9^{\prime \prime}$ or $\sim 0.2 \mathrm{pc}$ in projection onto the plane of the sky. The survey achieved a median sensitivity per spectral channel of $0.8 \mathrm{mJy}^{\text {beam }}{ }^{-1}$ (rms), with values ranging between $0.35 \mathrm{mJy}^{\text {beam }}{ }^{-1}$ and $1.1 \mathrm{mJy}^{\text {beam }}{ }^{-1}$.

Our search for acrylamide in Sgr B2(N) followed the same strategy as our search for propiolamide, $\mathrm{HCCC}(\mathrm{O}) \mathrm{NH}_{2}$, reported in Alonso et al. (2021). We focused the search toward the following two positions: the offset position Sgr B2(N1S) located at $(\alpha, \delta)_{\mathrm{J} 2000}=\left(17^{\mathrm{h}} 47^{\mathrm{m}} 19^{\mathrm{s}} .870,-28^{\circ} 22^{\prime} 19^{\prime} .48\right)$ and the secondary hot core Sgr B2(N2) at $(\alpha, \delta)_{\mathrm{J} 2000}=\left(17^{\mathrm{h}} 47^{\mathrm{m}} 19^{\mathrm{s}} .863\right.$, $-28^{\circ} 22^{\prime} 13^{\prime \prime}$.27). The former position was chosen by Belloche et al. (2019). With its location about $1^{\prime \prime}$ to the South of the main hot core Sgr B2(N1), its continuum emission has a lower opacity, which allows us to look deeper into the molecular content of Sgr B2(N1). Compared to Belloche et al. (2019), we employed a more recent version of our data set for which we have improved the splitting of the continuum and line emission as reported in Melosso et al. (2020).

We modeled the spectra of Sgr B2(N1S) and Sgr B2(N2) with the software Weeds (Maret et al. 2011) under the assumption of local thermodynamic equilibrium (LTE), which is appropriate given the high densities that characterize Sgr B2(N)'s hot cores $\left(>1 \times 10^{7} \mathrm{~cm}^{-3}\right.$, see Bonfand et al. 2019). A best-fit synthetic spectrum of each molecule was derived separately, and then the contributions of all identified molecules were added together. Each species was modeled with a set of five parameters: size of the emitting region $\left(\theta_{\mathrm{s}}\right)$, column density $(N)$, temperature $\left(T_{\text {rot }}\right)$, linewidth $(\Delta V)$, and velocity offset $\left(V_{\text {off }}\right)$ with respect to the assumed systemic velocity of the source, $V_{\text {sys }}=62 \mathrm{~km} \mathrm{~s}^{-1}$ for Sgr B2(N1S) and $V_{\text {sys }}=74 \mathrm{~km} \mathrm{~s}^{-1}$ for Sgr B2(N2).

\subsection{Nondetection of acrylamide}

To guide our search for the syn conformer of acrylamide in the ReMoCA spectra of Sgr B2(N1S) and Sgr B2(N2), we computed
LTE synthetic spectra of this molecule on the basis of the parameters derived for acetamide, $\mathrm{CH}_{3} \mathrm{C}(\mathrm{O}) \mathrm{NH}_{2}$, by Belloche et al. (2019) and Belloche et al. (2017), respectively. We kept only the column density of acrylamide as a free parameter. We searched for rotational lines in its vibrational ground state, $v=0$, and in its vibrationally excited states $v_{24}=1$ and $v_{24}=2$. No evidence for emission of acrylamide was found toward either source. The nondetection toward Sgr B2(N1S) and Sgr B2(N2) is illustrated in Figs. B.1-B.4, respectively, and the upper limits to the column density of acrylamide are indicated in Tables 4 and 5, respectively. The tables also recall the parameters that we previously obtained for acetamide and propiolamide. We did not take into account the contribution of the skew conformer of acrylamide to its partition function. This conformer is observed as a tunneling doublet about $540 \mathrm{~cm}^{-1}$ above the syn form (see Sect.3.2). Relative abundances of each conformer in Table 2 show that it contributes less than $3 \%$ to the partition function at 160-180 K, which does not have a significant impact on the upper limits reported for acrylamide in Tables 4 and 5.

\subsection{Nondetection of propionamide}

We also searched for propionamide, $\mathrm{C}_{2} \mathrm{H}_{5} \mathrm{C}(\mathrm{O}) \mathrm{NH}_{2}$, which has a fully saturated alkyl group and is structurally related to acrylamide. We used the spectroscopic predictions for its vibrational ground state, $v=0$, and its first vibrationally excited state, $v_{30}=$ 1, published in Li et al. (2021). We did not find any evidence for this molecule toward either Sgr B2(N1S) or Sgr B2(N2), as illustrated in Figs. B.5-B.8, respectively. Tables 4 and 5, respectively, report the upper limits that we derived for its column density.

Li et al. (2021) recently claimed the tentative detection of propionamide on the basis of the ReMoCA data set, which they downloaded from the ALMA archive and reduced themselves, toward a position located to the East of Sgr B2(N1) at $(\alpha, \delta)_{\mathrm{J} 2000}=$ $\left(17^{\mathrm{h}} 47^{\mathrm{m}} 19^{\mathrm{s}} 99,-28^{\circ} 22^{\prime} 18^{\prime \prime} \cdot 72\right)$. This position corresponds to an equatorial coordinate offset of $\left(1.58^{\prime \prime},-2.72^{\prime \prime}\right)$ with respect to the phase center. We also searched for propionamide toward this position using our own reduced version of the ReMoCA survey. We computed LTE synthetic spectra assuming the same source size, temperature, linewidth, and velocity as in Li et al. (2021), keeping only the column density as a free parameter. We did not find any evidence for propionamide toward this position either. The nondetection toward this position is illustrated in Figs. B.9 and B.10 and the upper limit to its column density is reported in Table 6. 
Table 5. Parameters of our best-fit LTE model of acetamide toward Sgr B2(N2), and upper limits for propiolamide, acrylamide, and propionamide.

\begin{tabular}{|c|c|c|c|c|c|c|c|c|c|c|}
\hline & Molecule & Status ${ }^{(a)}$ & $N_{\text {det }}{ }^{(b)}$ & $\begin{array}{c}\theta_{\mathrm{s}}{ }^{(c)} \\
\left({ }^{\prime \prime}\right)\end{array}$ & $\begin{array}{c}T_{\text {rot }}{ }^{(d)} \\
(\mathrm{K})\end{array}$ & $\begin{array}{c}N^{(e)} \\
\left(\mathrm{cm}^{-2}\right)\end{array}$ & $F_{\mathrm{vib}}(f)$ & $\begin{array}{c}\Delta V^{(g)} \\
\left(\mathrm{km} \mathrm{s}^{-1}\right)\end{array}$ & $\begin{array}{c}V_{\text {off }}{ }^{(h)} \\
\left(\mathrm{km} \mathrm{s}^{-1}\right)\end{array}$ & $\frac{N_{\mathrm{ref}}}{N}(i)$ \\
\hline Acetamide & $\mathrm{CH}_{3} \mathrm{C}(\mathrm{O}) \mathrm{NH}_{2}{ }^{(j) \star}$ & $\mathrm{d}$ & 23 & 0.9 & 180 & $1.4(17)$ & 1.23 & 5.0 & 1.5 & 1 \\
\hline Propiolamide & $\mathrm{HCCC}(\mathrm{O}) \mathrm{NH}_{2}{ }^{(k)}$ & $\mathrm{n}$ & 0 & 0.9 & 180 & $<1.0(16)$ & 1.30 & 5.0 & 0.0 & $>13$ \\
\hline Acrylamide & syn- $\mathrm{C}_{2} \mathrm{H}_{3} \mathrm{C}(\mathrm{O}) \mathrm{NH}_{2}$ & $\mathrm{n}$ & 0 & 0.9 & 180 & $<2.1(16)$ & 2.67 & 5.0 & 0.0 & $>6.3$ \\
\hline Propionamide & $\mathrm{C}_{2} \mathrm{H}_{5} \mathrm{C}(\mathrm{O}) \mathrm{NH}_{2}$ & $\mathrm{n}$ & 0 & 0.9 & 180 & $<4.3(16)$ & 5.06 & 5.0 & 0.0 & $>3.1$ \\
\hline
\end{tabular}

Notes. ${ }^{(a)}$ d: detection, n: nondetection. ${ }^{(b)}$ Number of detected lines (conservative estimate, see Sect. 3 of Belloche et al. 2016). One line of a given species may mean a group of transitions of that species that are blended together. ${ }^{(c)}$ Source diameter (FWHM). ${ }^{(d)}$ Rotational temperature. ${ }^{(e)}$ Total column density of the molecule. $x(y)$ means $x \times 10^{y} .{ }^{(f)}$ Correction factor that was applied to the column density to account for the contribution of vibrationally excited states, in the cases where this contribution was not included in the partition function of the spectroscopic predictions. ${ }^{(g)}$ Linewidth (FWHM). ${ }^{(h)}$ Velocity offset with respect to the assumed systemic velocity of Sgr B2(N2), $V_{\text {sys }}=74 \mathrm{~km} \mathrm{~s}^{-1}$. ${ }^{(i)}$ Column density ratio, with $N_{\text {ref }}$ the column density of the previous reference species marked with a $\star{ }^{(j)}$ The parameters were derived from the EMoCA survey by Belloche et al. (2017). ${ }^{(k)}$ The upper limit was derived from the ReMoCA survey by Alonso et al. (2021).

Table 6. Column density upper limit derived for propionamide toward the offset position $\left(1.58^{\prime \prime},-2.72^{\prime \prime}\right)$ with respect to the phase center.

\begin{tabular}{ccccccccc}
\hline \hline Molecule & Status ${ }^{(a)}$ & $N_{\text {det }}{ }^{(b)}$ & $\begin{array}{c}\theta_{\mathrm{s}}{ }^{(c)} \\
\left({ }^{\prime \prime}\right)\end{array}$ & $\begin{array}{c}T_{\text {rot }}{ }^{(d)} \\
(\mathrm{K})\end{array}$ & $\begin{array}{c}N^{(e)} \\
\left(\mathrm{cm}^{-2}\right)\end{array}$ & $F_{\text {vib }}{ }^{(f)}$ & $\begin{array}{c}\Delta V^{(g)} \\
\left(\mathrm{km} \mathrm{s}^{-1}\right)\end{array}$ & $\begin{array}{c}V_{\text {off }}{ }^{(h)} \\
\left(\mathrm{km} \mathrm{s}^{-1}\right)\end{array}$ \\
\hline $\mathrm{C}_{2} \mathrm{H}_{5} \mathrm{C}(\mathrm{O}) \mathrm{NH}_{2}$ & $\mathrm{n}$ & 0 & 2.3 & 150 & $<1.3(16)$ & 3.77 & 4.2 & 0.0 \\
\hline
\end{tabular}

Notes. ${ }^{(a)}$ n: nondetection. ${ }^{(b)}$ Number of detected lines. ${ }^{(c)}$ Source diameter (FWHM). ${ }^{(d)}$ Rotational temperature. ${ }^{(e)}$ Total column density of the molecule. $x(y)$ means $x \times 10^{y} .{ }^{(f)}$ Correction factor that was applied to the column density to account for the contribution of vibrationally excited states, in the cases where this contribution was not included in the partition function of the spectroscopic predictions. ${ }^{(g)}$ Linewidth (FWHM). ${ }^{(h)}$ Velocity offset with respect to the assumed systemic velocity, $V_{\text {sys }}=63 \mathrm{~km} \mathrm{~s}^{-1}$.

\section{Discussion}

\subsection{Laboratory spectroscopy of acrylamide}

The spectroscopic results from Sect. 3 represent a substantial improvement in the laboratory characterization of the rotational spectrum of acrylamide. We have significantly extended the frequency coverage with over 8500 new transitions assigned and analyzed. The derived spectroscopic constants reproduce the assigned transitions within the experimental uncertainties on average and allow us to confidently predict the rotational transitions of acrylamide over a broad range of frequencies. Of particular interest in this work was the observational window from 84.1 to $114.4 \mathrm{GHz}$ in which the rotational transitions already revealed significant differences between the experimental frequencies and spectral predictions based on the original microwave work of Marstokk et al. (2000). For example, ground state $K_{a}=1 \leftarrow 0$ transitions of the syn conformer were shifted by almost $2 \mathrm{MHz}$ at the predicted uncertainty around $100 \mathrm{kHz}$. Since the syn conformer represents the global minimum on the potential energy landscape of acrylamide, it is the most relevant species for observational purposes. Its ground state catalog derived from this work represents a highly accurate observational reference and will be available in the Cologne Database for Molecular Spectroscopy (CDMS) to support future searches for acrylamide in space, for instance, in warm or lukewarm environments.

A comparison between the derived spectroscopic parameters of the syn conformer and those from the previous microwave study is shown in Table 1. The most immediate conclusion to be extracted from this table is that the accuracy of these parameters is significantly improved for both the ground state and the two low-lying excited vibrational states. However, it is noticeable that while the rotational and quartic centrifugal distortion constants agree with those from Marstokk et al. (2000), significant discrepancies are found for the sextic constants. These discrepancies are very likely attributed to incorrectly treated nuclear quadrupole hyperfine splitting and, to a lesser extent, to a few erroneous assignments in the earlier study. It has been revealed that many transitions need to be assigned to frequencies of the overlapping $F=J \pm 1$ hyperfine components, instead of the hyperfine-free frequencies. Rather large differences in observed minus calculated frequencies for these transitions were then artificially fit to sextic centrifugal distortion constants. However, this is no longer possible when a significantly larger data set is combined with these transitions; they clearly appear as outliers in the fit. Taking the syn conformer ground state as an example, Table A.3 shows that when the incriminated lines are removed from the data set of Marstokk et al. (2000), the remaining transitions can be well fitted using two sextic constants. Their values are close to their counterparts determined solely from the data set measured in this work. Table A.3. also shows that the microwave data below $60 \mathrm{GHz}$ have no impact on the results of the global fit presented here. It can be explained by the fact that the access of quantum numbers of the present and previous data sets are similar. Nevertheless, the low-frequency data are maintained in the global fit for completeness.

An interesting phenomenon seen in this study is the tunneling splitting for the skew conformer that has been observed for this species for the first time. The results of the fit for the ground state tunneling doublet are provided in Table 3 alongside the results of the previous work. In that work, the two states were analyzed separately and were assigned to the ground state (here $0^{+}$) and the excited $\mathrm{C}-\mathrm{C}$ torsional state (here $0^{-}$). The spectroscopic constants are significantly improved in terms of 
accuracy and are consistent with those reported previously. Differences are attributable to substantially larger data set treated in this work, containing a variety of transitions. For example, only $14 a$-type $R$-branch transitions were measured for the $0^{-}$ state by Marstokk et al. (2000) while over 1200 lines encompassing $a$-type and $b$-type $R$-branch and $b$-type $Q$-branch transitions were measured here. Although this conformer is less important for observational purposes, we made a considerable progress in the characterization of its rotational spectrum and deliver first experimental information on tunneling splitting for this species.

\subsection{Comparison to other molecules in $\operatorname{Sgr} B 2(N)$}

The nondetection of acrylamide reported in Sect. 4.2 implies that acrylamide is at least $\sim 26$ and $\sim 6$ times less abundant than acetamide toward Sgr B2(N1S) and Sgr B2(N2), respectively. For comparison, vinyl cyanide, $\mathrm{C}_{2} \mathrm{H}_{3} \mathrm{CN}$, is about five times less abundant than methyl cyanide, $\mathrm{CH}_{3} \mathrm{CN}$, toward Sgr B2(N2) (Belloche et al. 2016) and a preliminary analysis of the ReMoCA survey yields a similar (slightly smaller) difference for Sgr B2(N1S). Both pairs of molecules, the amides $\mathrm{C}_{2} \mathrm{H}_{3} \mathrm{C}(\mathrm{O}) \mathrm{NH}_{2} / \mathrm{CH}_{3} \mathrm{C}(\mathrm{O}) \mathrm{NH}_{2}$ and the cyanides $\mathrm{C}_{2} \mathrm{H}_{3} \mathrm{CN} / \mathrm{CH}_{3} \mathrm{CN}$, share the same structural difference (an unsaturated $\mathrm{C}_{2} \mathrm{H}_{3}$ group replaced with a saturated $\mathrm{CH}_{3}$ group). The upper limit to the abundance ratio $\mathrm{C}_{2} \mathrm{H}_{3} \mathrm{C}(\mathrm{O}) \mathrm{NH}_{2} / \mathrm{CH}_{3} \mathrm{C}(\mathrm{O}) \mathrm{NH}_{2}$ toward Sgr B2(N2) is not constraining compared to the cyanide ratio, but toward Sgr B2(N1S), the amide ratio is at least five times smaller than the cyanide ratio.

\subsection{Nondetection of propionamide}

Some of the ALMA spectra shown in Figs. B.9 and B.10 differ from the spectra shown in Figs. 5-7 of Li et al. (2021). At least two reasons can explain these differences: first, Belloche et al. (2019) performed several iterations of self-calibration when reducing the interferometric data that were used here, while $\mathrm{Li}$ et al. (2021) probably did not; second, and more importantly, the determination of the continuum level is notoriously difficult in spectra close to the confusion limit. The automatic algorithm that we used to fit the continuum level for each pixel of the data cube relies on the distribution of intensities of each pixel and removes a zeroth-order baseline (see Sect. 2.2 of Belloche et al. 2019). In contrast, Li et al. (2021) fitted first-order baselines after a manual selection of 3-5 groups of channels that seemed to be free of line emission. These different approaches can lead in some cases to different fitted continuum levels. This illustrates the additional uncertainty that arises from the continuum fitting and affects the position of the level used to assess the significance of a line detection (e.g., the $3 \sigma$ level in the figures shown in Appendix B).

Several portions of the ReMoCA spectrum where we find discrepancies between the synthetic and observed spectra were not shown in Figs. 5-7 of Li et al. (2021), for instance the transitions at 91 376, 93 432, 97320,99288 , or $102568 \mathrm{MHz}$ in Fig. B.9, 94030 or 99739 MHz in Fig. B.10. In addition, some of the lines of propionamide claimed by Li et al. (2021) as clearly detected in their Fig. 5 cannot be considered as detected in our spectra, for instance those at 92 403, 92 408, or $103342 \mathrm{MHz}$. Finally, Table 4 indicates that propionamide is at least a factor 14 times less abundant than acetamide toward Sgr B2(N1S). It would be surprising if propionamide would be nearly as abundant as acetamide at the position offset to the East as claimed by $\mathrm{Li}$ et al. (2021). We conclude from this that there is so far no convincing evidence for the presence of propionamide in the interstellar medium.

\subsection{Formation mechanisms for acrylamide and related species}

In Alonso et al. (2021) we discussed the possible formation mechanisms for propiolamide in light of its nondetection, guided by findings from recent astrochemical kinetics models. Even though there are no chemical networks at present that would appear to include either propiolamide, acrylamide, or propionamide, the behavior of related molecules can provide some insight into their plausible characteristics. Alonso et al. (2021) proposed that propiolamide could be formed on grain surfaces at early times in the evolution of a hot core, when the gas and dust are cold and the dust-grain ice mantles are just beginning to form. At that point in time, small unsaturated hydrocarbons should be relatively abundant in the gas phase; from there, some fraction would be deposited onto the grain surfaces, where it could react further with other species. The adsorption onto the grains of $\mathrm{C}_{2} \mathrm{H}$ in particular, or its production from other species adsorbed from the gas phase, could lead to reaction with the radical $\mathrm{NH}_{2} \mathrm{CO}$ to form propiolamide. Alternatively, $\mathrm{C}_{2} \mathrm{H}$ could react with mobile atomic $\mathrm{H}$, to produce the more stable acetylene, $\mathrm{C}_{2} \mathrm{H}_{2}$. The production on the grains of $\mathrm{CH}_{3}$ could likewise lead to a reaction with $\mathrm{NH}_{2} \mathrm{CO}$ to produce acetamide, with the main alternative being hydrogenation to $\mathrm{CH}_{4}$. Molecules formed on the grains in this way at low temperatures would ultimately be released into the gas phase during the later hot stage of evolution.

With this in mind, Alonso et al. (2021) used the relative solidphase abundances of $\mathrm{C}_{2} \mathrm{H}_{2}$ and $\mathrm{CH}_{4}$ obtained in the models of Garrod et al. (2022) to infer the possible ratio of propiolamide to acetamide on the grains. Here, we extend this analysis to consider also acrylamide and propionamide, using $\mathrm{C}_{2} \mathrm{H}_{4}$ and $\mathrm{C}_{2} \mathrm{H}_{6}$ as proxies, respectively. As before, values are taken from the endtime of the cold collapse stage of evolution used in the models (technically, the final model setup of Garrod et al. 2022). We find solid-phase ratios of $\mathrm{CH}_{4}$ with respect to $\mathrm{C}_{2} \mathrm{H}_{2}, \mathrm{C}_{2} \mathrm{H}_{4}$, and $\mathrm{C}_{2} \mathrm{H}_{6}$ of 2800 , 440, and 27 , respectively. This suggests that if formed through cold addition of $\mathrm{C}_{2} \mathrm{H}_{3}$ to $\mathrm{NH}_{2} \mathrm{CO}$ on grain surfaces, acrylamide should be around 440 times less abundant than acetamide. This value is in nominal agreement with the upper limit values obtained for Sgr B2(N1S) and Sgr B2(N2), and suggests that this molecule could have an abundance at least an order of magnitude below those limits. We note, however, that the ratios from the models do not consider any formation or destruction that could occur during the hot stage, nor do they take account of differences in binding energies or any associated variations in spatial arrangement in the gas phase, following thermal release from the grains.

Interestingly, the inferred ratio of acetamide to propionamide is only 27 , which is indeed only a factor of two greater than the observational limit toward Sgr B2(N1S), while it is consistent with the nondetection in each source. We note also that the claim of Li et al. (2021) that propionamide is detected in Sgr B2(N) with an abundance nearly as high as acetamide is inconsistent with the abundance ratio that we infer for these species from the models.

As noted by Alonso et al. (2021), propiolamide (formed in whatever fashion) could be further hydrogenated on the dust grains all the way to propionamide. Two necessary steps in this process would be the production, and then destruction, of acrylamide, through the addition of atomic H. However, abstraction of $\mathrm{H}$ from any of these species or their intermediates through alternative reaction pathways with mobile atomic $\mathrm{H}$ could result in conversion in the other direction. Depending on the balance 
of activation energy barriers and branching ratios, this could therefore lead to some stable ratio between acrylamide and the other two. In fact, something similar to this situation occurs in the astrochemical models for the species $\mathrm{C}_{2} \mathrm{H}_{2}, \mathrm{C}_{2} \mathrm{H}_{4}$, and $\mathrm{C}_{2} \mathrm{H}_{6}$ as well. We should therefore expect that the ratios derived above would already reflect this sort of relationship (any dissimilarities in activation energy barriers notwithstanding).

Alternative mechanisms for producing propiolamide, acrylamide, and propionamide, such as $\mathrm{NH}_{2}$ addition to the radical $\mathrm{C}_{2} \mathrm{H}_{3} \mathrm{CO}$, are more difficult to assess based on inference from the models, but they also appear plausible. Another alternative mechanism for propionamide formation would be a more direct conversion of acetamide, via the abstraction of an $\mathrm{H}$ atom from the methyl group (by $\mathrm{H}$ or some reactive radical such as $\mathrm{OH}$ ), followed by the addition of another methyl group. Garrod et al. (2022) also invoked the reaction of methylene $\left(\mathrm{CH}_{2}\right)$ with methanol $\left(\mathrm{CH}_{3} \mathrm{OH}\right)$ on the grains, to produce ethanol $\left(\mathrm{C}_{2} \mathrm{H}_{5} \mathrm{OH}\right)$ as well as other products. Methylene reactions might provide a route to lengthen alkyl groups in other molecules, including acetamide. This could suggest perhaps an even greater abundance for propionamide, by some unknown degree.

A more rugged test of these ideas would involve the development of a self-consistent network for all of the above species, as well as for related alkyl-group bearing molecules whose production may be competitive with the others. Application of the network to the full hot-core physical treatment would then be possible.

\section{Conclusions}

In the present work we conducted a detailed rotational study of acrylamide, a peptide bond bearing species, from 75 to $480 \mathrm{GHz}$. More than 6000 new rotational lines for the ground state and two excited vibrational states were measured and assigned for the most stable syn conformer. In addition to this, over 2500 lines were analyzed for the less stable skew conformer. The ground vibrational state of this conformer was observed in the form of a doublet. We interpreted this observation as a consequence of a double-minimum potential function with a small barrier between two equivalent skew structures. A comprehensive spectral analysis provided accurate frequency predictions to search for acrylamide in Sgr B2(N). Additionally, emission lines from the related molecule propionamide were searched for in the same source. The main conclusions of these searches are following:

1. Acrylamide was not detected toward the hot molecule cores Sgr B2(N1S) and Sgr B2(N2) with ALMA. The upper limits derived for its column density imply that it is at least 26 and 6 times less abundant than acetamide toward these sources, respectively.

2. Propionamide was not detected toward Sgr B2(N1S) and Sgr B2(N2) either. It is at least 14 and 3 times less abundant than acetamide toward these sources, respectively.

3. We do not confirm either the tentative detection of propionamide recently reported in the literature toward a position offset to the East of Sgr B2(N1). We conclude from this that there is so far no convincing evidence to support the presence of propionamide in the interstellar medium.

While acrylamide is not yet present in any astrochemical kinetics models, comparisons with model results for related species suggest that acrylamide may be a few hundred times less abundant than acetamide, corresponding to a value at least an order of magnitude lower than the observational upper limits.
Propionamide may be as little as a factor of two less abundant than the upper limit toward Sgr B2(N1S).

Acknowledgements. This work has been supported by the Czech Science Foundation (GACR, grant 19-25116Y). The funding is gratefully acknowledged. We thank Roman Motiyenko and Laurent Margulès for sending us spectroscopic predictions for propionamide in electronic format. This paper makes use of the following ALMA data: ADS/JAO.ALMA\#2016.1.00074.S. ALMA is a partnership of ESO (representing its member states), NSF (USA), and NINS (Japan), together with NRC (Canada), NSC and ASIAA (Taiwan), and KASI (Republic of Korea), in cooperation with the Republic of Chile. The Joint ALMA Observatory is operated by ESO, AUI/NRAO, and NAOJ. The interferometric data are available in the ALMA archive at https://almascience.eso.org/aq/. Part of this work has been carried out within the Collaborative Research Centre 956, sub-project B3, funded by the Deutsche Forschungsgemeinschaft (DFG) project ID 184018867. R.T.G. acknowledges support from the National Science Foundation (grant No. AST 19-06489).

\section{References}

Alonso, E. R., Kolesniková, L., Belloche, A., et al. 2021, A\&A, 647, A55 Barone, V., Latouche, C., Skouteris, D., et al. 2015, MNRAS, 453, L31 Belloche, A., Menten, K. M., Comito, C., et al. 2008, A\&A, 482, 179 Belloche, A., Müller, H. S. P., Menten, K. M., Schilke, P., \& Comito, C. 2013, A\&A, 559, A47

Belloche, A., Müller, H. S. P., Garrod, R. T., \& Menten, K. M. 2016, A\&A, 587, A91

Belloche, A., Meshcheryakov, A. A., Garrod, R. T., et al. 2017, A\&A, 601, A49 Belloche, A., Garrod, R. T., Müller, H. S. P., et al. 2019, A\&A, 628, A10 Bonfand, M., Belloche, A., Garrod, R. T., et al. 2019, A\&A, 628, A27 Cernicharo, J., Agúndez, M., Cabezas, C., et al. 2021, A\&A, 647, L2 Christen, D., \& Müller, H. S. P. 2003, Phys. Chem. Chem. Phys., 5, 3600 Colzi, L., Rivilla, V. M., Beltrán, M. T., et al. 2021, A\&A, 653, A129

Coutens, A., Jørgensen, J. K., van der Wiel, M. H. D., et al. 2016, A\&A, 590, L6

Daly, A., Kolesniková, L., Mata, S., \& Alonso, J. 2014, J. Mol. Spectr., 306, 11 Duarte, A., Amorim da Costa, A., \& Amado, A. 2005, J. Mol. Struct., 723, 63 Frenkel-Pinter, M., Samanta, M., Ashkenasy, G., \& Leman, L. J. 2020, Chem. Rev., 120, 4707

Frigge, R., Zhu, C., Turner, A. M., et al. 2018, ApJ, 862, 84

Frisch, M. J., Trucks, G. W., Schlegel, H. B., et al. 2016, Gaussian16 Revision B.01 (Gaussian Inc: Wallingford CT)

Gardner, F. F., \& Winnewisser, G. 1975, ApJ, 195, L127

Garrod, R. T., Jin, M., Matis, K. A., et al. 2022, ApJS, 259, 1

Gorai, P., Bhat, B., Sil, M., et al. 2020, ApJ, 895, 86

Gordy, W., \& Cook, R. L. 1970, Microwave Molecular Spectra (Geneva: Interscience Publishers)

Gottlieb, C. A., Palmer, P., Rickard, L. J., \& Zuckerman, B. 1973, ApJ, 182, 699

Halfen, D. T., Ilyushin, V., \& Ziurys, L. M. 2011, ApJ, 743, 60

Herbst, E., \& van Dishoeck, E. F. 2009, ARA\&A, 47, 427

Hollis, J. M., Lovas, F. J., \& Jewell, P. R. 2000, ApJ, 540, L107

Hollis, J. M., Jewell, P. R., Lovas, F. J., Remijan, A., \& Møllendal, H. 2004, ApJ, 610, L21

Hollis, J. M., Lovas, F. J., Remijan, A. J., et al. 2006, ApJ, 643, L25

Jørgensen, J. K., Belloche, A., \& Garrod, R. T. 2020, ARA\&A, 58, 727

Kahane, C., Ceccarelli, C., Faure, A., \& Caux, E. 2013, ApJ, 763, L38

Kaiser, R. I., Stockton, A. M., Kim, Y. S., Jensen, E. C., \& Mathies, R. A. 2013 ApJ, 765, 111

Kania, P., Stř́iteská, L., Šimečková, M., \& Š. Urban. 2006, J. Mol. Struct., 795, 209

Kisiel, Z., Pszczółkowski, L., Medvedev, I. R., et al. 2005, J. Mol. Spectr., 233, 231

Kisiel, Z., Pszczółkowski, L., Drouin, B. J., et al. 2012, J. Mol. Spectr., 280, 134

Kisiel, Z., Kraśnicki, A., Jabs, W., et al. 2013, J. Phys. Chem. A, 117, 9889

Kisiel, Z., Kolesniková, L., Belloche, A., et al. 2022, A\&A, 657, A99

Koucký, J., Kania, P., Uhlíková, T., et al. 2013, J. Phys. Chem. A, 117, 10138

Kydd, R., \& Dunham, A. 1980, J. Mol. Struct., 69, 79

Lee, K. L. K., Loomis, R. A., Burkhardt, A. M., et al. 2021, ApJ, 908, L11

Li, J., Wang, J., Lu, X., et al. 2021, ApJ, 919, 4

Ligterink, N. F. W., Terwisscha van Scheltinga, J., Taquet, V., et al. 2018, MNRAS, 480, 3628

Ligterink, N. F. W., El-Abd, S. J., Brogan, C. L., et al. 2020, ApJ, 901, 37

Ligterink, N. F. W., Ahmadi, A., Coutens, A., et al. 2021, A\&A, 647, A87

López-Sepulcre, A., Balucani, N., Ceccarelli, C., et al. 2019, ACS Earth Space Chem., 3, 2122 
Marcelino, N., Cernicharo, J., Agúndez, M., et al. 2007, ApJ, 665, L127

Maret, S., Hily-Blant, P., Pety, J., Bardeau, S., \& Reynier, E. 2011, A\&A, 526, A47

Margulès, L., McGuire, B. A., Senent, M. L., et al. 2017, A\&A, 601, A50

Marstokk, K.-M., Møllendal, H., \& Samdal, S. 2000, J. Mol. Struct., 524, 69

McGuire, B. A. 2018, ApJS, 239, 17

Melosso, M., Belloche, A., Martin-Drumel, M.-A., et al. 2020, A\&A, 641, A160

Mendoza, E., Lefloch, B., López-Sepulcre, A., et al. 2014, MNRAS, 445, 151

Motiyenko, R. A., Tercero, B., Cernicharo, J., \& Margulès, L. 2012, A\&A, 548, A71

Motiyenko, R. A., Margules, L., Despois, D., \& Guillemin, J.-C. 2018, Phys Chem. Chem. Phys., 20, 5509

Nummelin, A., Bergman, P., Hjalmarson, A., et al. 1998, ApJS, 117, 427

Pickett, H. M. 1972, J. Chem. Phys., 56, 1715

Pickett, H. M. 1991, J. Mol. Spectr., 148, 371

Quénard, D., Jiménez-Serra, I., Viti, S., Holdship, J., \& Coutens, A. 2018 MNRAS, 474, 2796

Redondo, P., Martínez, H., Cimas, A., Barrientos, C., \& Largo, A. 2013, Phys. Chem. Chem. Phys., 15, 13005

Redondo, P., Barrientos, C., \& Largo, A. 2014, ApJ, 793, 32

Reid, M. J., Menten, K. M., Brunthaler, A., et al. 2019, ApJ, 885, 131
Rimola, A., Skouteris, D., Balucani, N., et al. 2018, ACS Earth Space Chem., 2, 720

Rivilla, V. M., Martín-Pintado, J., Jiménez-Serra, I., et al. 2020, ApJ, 899, L28

Rivilla, V. M., Jiménez-Serra, I., Martín-Pintado, J., et al. 2021, PNAS, 118, e2101314118

Rubin, R., G.W. Swenson, J., Benson, R., Tigelaar, H., \& Flygare, W. 1971, ApJ, 169, L39

Ruiz-Mirazo, K., Briones, C., \& de la Escosura, A. 2014, Chem. Rev., 114, 285

Sandford, S. A., Nuevo, M., Bera, P. P., \& Lee, T. J. 2020, Chem. Rev., 120, 4616

Sanz-Novo, M., Belloche, A., Alonso, J. L., et al. 2020, A\&A, 639, A135

Stolar, T., Grubešić, S., Cindro, N., et al. 2021, Angew. Chem. Int. Ed., 60, 12727

Tercero, F., López-Pérez, J. A., Gallego, J. D., et al. 2021, A\&A, 645, A37

Thiel, V., Belloche, A., Menten, K. M., Garrod, R. T., \& Müller, H. S. P. 2017, A\&A, 605, L6

Turner, B. E. 1989, ApJS, 70, 539

Turner, B. E., \& Apponi, A. J. 2001, ApJ, 561, L207

Urban, S., \& Sarka, K. 1990, J. Mol. Spectr., 144, 446

Watson, J. K. G. 1977, in Vibrational Spectra and Structure, ed. J. R. Durig (Amsterdam: Elsevier), 6, 1

Weber, A. L., \& Pizzarello, S. 2006, PNAS, 103, 12713

Zingsheim, O., Müller, H. S., Lewen, F., Jørgensen, J. K., \& Schlemmer, S. 2017, J. Mol. Spectr., 342, 125 


\section{Appendix A: Complementary tables}

Table A.1 lists the measured transitions of syn acrylamide. Table A.2 lists the frequencies of normal vibrational modes of syn and skew acrylamide used to calculate the vibrational partition function. Table A.3 lists a comparison of the ground state spectroscopic constants of syn acrylamide obtained from different data sets. Table A.4 lists the measured transitions of skew acrylamide.

\section{Appendix B: Complementary figures: \\ Astronomical spectra}

Figures B.1-B.4 illustrate the nondetection of acrylamide in its ground state, $v=0$, and its first vibrationally excited state, $v_{24}=1$, toward Sgr B2(N1S) and Sgr B2(N2). Figures B.5-B.8 illustrate the nondetection of propionamide in its ground state, $v=0$, and its first vibrationally excited state, $v_{30}=1$, toward Sgr B2(N1S) and Sgr B2(N2). Figures B.9 and B.10 illustrate the nondetection of propionamide in its ground state, $v=0$, and its first vibrationally excited state, $v_{30}=1$, toward the equatorial offset position $\left(1.58^{\prime \prime},-2.72^{\prime \prime}\right)$ relative to the phase center. 
L. Kolesniková et al.: The rotational spectrum and ISM search of acrylamide

Table A.1. List of the measured transitions of syn acrylamide.

\begin{tabular}{|c|c|c|c|c|c|c|c|c|c|c|c|c|}
\hline Vib. state & $J^{\prime}$ & $K_{a}^{\prime}$ & $K_{c}^{\prime}$ & $J^{\prime \prime}$ & $K_{a}^{\prime \prime}$ & $K_{c}^{\prime \prime}$ & $v_{\mathrm{obs}}(\mathrm{MHz})^{a}$ & $v_{\mathrm{obs}}-v_{\mathrm{calc}}(\mathrm{MHz})^{b}$ & $u_{\mathrm{obs}}(\mathrm{MHz})^{c}$ & $\left(v_{\mathrm{obs}}-v_{\text {calc }}\right)_{\text {blends }}(\mathrm{MHz})^{d}$ & Weight $^{e}$ & Notes $^{f}$ \\
\hline G.S. & 15 & 4 & 11 & 15 & 3 & 12 & 38737.1700 & 0.0844 & 0.100 & & & (1) \\
\hline G.S. & 13 & 2 & 12 & 12 & 1 & 11 & 88541.9842 & 0.0120 & 0.020 & & & (2) \\
\hline G.S. & 16 & 1 & 16 & 15 & 0 & 15 & 100009.6024 & 0.0088 & 0.020 & & & (2) \\
\hline G.S. & 32 & 3 & 30 & 31 & 2 & 29 & 209078.0213 & -0.0381 & 0.020 & 0.0126 & 0.50 & (2) \\
\hline G.S. & 32 & 2 & 30 & 31 & 3 & 29 & 209078.0213 & 0.0632 & 0.020 & 0.0126 & 0.50 & (2) \\
\hline$v_{24}=1$ & 14 & 2 & 12 & 13 & 3 & 11 & 96383.9981 & -0.0071 & 0.020 & & & (2) \\
\hline$v_{24}=1$ & 41 & 3 & 38 & 40 & 4 & 37 & 270039.3106 & 0.0019 & 0.020 & -0.0009 & 0.50 & (2) \\
\hline$v_{24}=1$ & 41 & 4 & 38 & 40 & 3 & 37 & 270039.3106 & -0.0038 & 0.020 & -0.0009 & 0.50 & (2) \\
\hline$v_{24}=2$ & 28 & 5 & 24 & 27 & 4 & 23 & 197984.2945 & 0.0226 & 0.020 & & & (2) \\
\hline$v_{24}=2$ & 65 & 9 & 56 & 64 & 10 & 55 & 452320.1788 & 0.0130 & 0.020 & -0.0192 & 0.50 & (2) \\
\hline$v_{24}=2$ & 65 & 10 & 56 & 64 & 9 & 55 & 452320.1788 & -0.0513 & 0.020 & -0.0192 & 0.50 & (2) \\
\hline
\end{tabular}

Notes. ${ }^{(a)}$ Observed frequency. ${ }^{(b)}$ Observed minus calculated frequency. ${ }^{(c)}$ Uncertainty of the observed frequency. ${ }^{(d)}$ Observed minus calculated frequency for blends. ${ }^{(e)}$ Intensity weighting factor for blended transitions. ${ }^{(f)}$ Source of the data: (1) Marstokk et al. (2000), (2) This work. This table is available in its entirety at the CDS.

Table A.2. Frequencies of normal vibrational modes of the two conformers of acrylamide used to calculate their vibrational partition function.

\begin{tabular}{rrrrrr}
\hline \hline & \multicolumn{2}{c}{ Syn conformer } & & \multicolumn{2}{c}{ Skew conformer } \\
\cline { 2 - 3 } \cline { 5 - 6 } Mode & Frequency $\left(\mathrm{cm}^{-1}\right)$ & Symmetry & & Frequency $\left(\mathrm{cm}^{-1}\right)$ & Symmetry \\
\hline 1 & 3727.6 & $A^{\prime}$ & 3749.7 & $A$ \\
2 & 3593.3 & $A^{\prime}$ & 3584.2 & $A$ \\
3 & 3236.1 & $A^{\prime}$ & 3219.3 & $A$ \\
4 & 3151.1 & $A^{\prime}$ & 3178.8 & $A$ \\
5 & 3140.0 & $A^{\prime}$ & 3137.9 & $A$ \\
6 & 1764.3 & $A^{\prime}$ & 1758.6 & $A$ \\
7 & 1689.6 & $A^{\prime}$ & 1684.8 & $A$ \\
8 & 1617.3 & $A^{\prime}$ & 1617.6 & $A$ \\
9 & 1443.8 & $A^{\prime}$ & 1455.7 & $A$ \\
10 & 1356.3 & $A^{\prime}$ & 1359.9 & $A$ \\
11 & 1290.0 & $A^{\prime}$ & 1311.6 & $A$ \\
12 & 1113.2 & $A^{\prime}$ & 1116.6 & $A$ \\
13 & 1037.9 & $A^{\prime}$ & 1043.9 & $A$ \\
14 & 811.5 & $A^{\prime}$ & 1040.3 & $A$ \\
15 & 616.8 & $A^{\prime}$ & 986.4 & $A$ \\
16 & 466.2 & $A^{\prime}$ & 824.1 & $A$ \\
17 & 276.9 & $A^{\prime}$ & 819.0 & $A$ \\
18 & 1018.8 & $A^{\prime \prime}$ & & 592.9 & $A$ \\
19 & 1008.6 & $A^{\prime \prime}$ & 561.3 & $A$ \\
20 & 821.2 & $A^{\prime \prime}$ & & 525.1 & $A$ \\
21 & 616.9 & $A^{\prime \prime}$ & 432.0 & $A$ \\
22 & 469.2 & $A^{\prime \prime}$ & 353.8 & $A$ \\
23 & 262.8 & $A^{\prime \prime}$ & & $A$ \\
24 & $90^{b}$ & $A^{\prime \prime}$ & & $A 9.9$ & $A$ \\
\hline
\end{tabular}

Notes. ${ }^{(a)}$ Taken from the IR measurements from Kydd \& Dunham (1980). ${ }^{(b)}$ From relative intensity measurements from Marstokk et al. (2000). The stated uncertainty is $10 \mathrm{~cm}^{-1}$. The rest of the vibrational modes is taken from the theoretical calculations at the B3LYP/cc-pVTZ level of theory performed by Marstokk et al. (2000). 
Table A.3. Comparison of the ground state spectroscopic constants of syn acrylamide obtained from different data sets $(A$-reduction, $\mathrm{I}^{\mathrm{r}}$-representation).

\begin{tabular}{|c|c|c|c|c|}
\hline & Data set I ${ }^{a}$ & Data set II ${ }^{b}$ & Data set III ${ }^{c}$ & Data set IV $^{d}$ \\
\hline$A / \mathrm{MHz}$ & $10732.8296(34)^{e}$ & $10732.8220(33)$ & $10732.819329(66)$ & $10732.819331(65)$ \\
\hline$B / \mathrm{MHz}$ & $4218.7012(13)$ & $4218.6933(11)$ & $4218.690258(41)$ & $4218.690256(41)$ \\
\hline $\mathrm{C} / \mathrm{MHz}$ & 3030.7434 (13) & 3030.7494 (23) & $3030.752982(33)$ & $3030.752979(33)$ \\
\hline$\Delta_{J} / \mathrm{kHz}$ & $0.7043(33)$ & $0.7396(61)$ & $0.786817(13)$ & $0.786816(13)$ \\
\hline$\Delta_{J K} / \mathrm{kHz}$ & $3.370(22)$ & 3.467 (44) & $3.755001(41)$ & 3.755014 (41) \\
\hline$\Delta_{K} / \mathrm{kHz}$ & $5.403(8)$ & $5.458(17)$ & $5.49543(11)$ & $5.49540(11)$ \\
\hline$\delta_{J} / \mathrm{kHz}$ & $0.2417(7)$ & 0.2249 (11) & $0.2227674(57)$ & $0.2227676(56)$ \\
\hline$\delta_{K} / \mathrm{kHz}$ & $3.20(4)$ & $3.315(81)$ & $3.361824(72)$ & $3.361833(72)$ \\
\hline$\Phi_{J} / \mathrm{mHz}$ & $-66(6)$ & $\ldots{ }^{f}$ & 0.1763 (16) & 0.1763 (16) \\
\hline$\Phi_{J K} / \mathrm{mHz}$ & $-222(34)$ & $\ldots{ }^{f}$ & 1.357 (16) & $1.361(16)$ \\
\hline$\Phi_{K J} / \mathrm{mHz}$ & $-1540(130)$ & $-23.17(58)$ & $-30.829(56)$ & $-30.836(56)$ \\
\hline$\Phi_{K} / \mathrm{mHz}$ & $570(40)$ & $54.8(10)$ & $55.102(77)$ & $55.087(76)$ \\
\hline$\phi_{J} / \mathrm{mHz}$ & $1.70(34)$ &...$^{f}$ & $0.09113(78)$ & 0.09118 (77) \\
\hline$\phi_{J K} / \mathrm{mHz}$ & $-600(50)$ &...$^{f}$ & $1.102(16)$ & $1.104(16)$ \\
\hline$\phi_{K} / \mathrm{mHz}$ & 1980 (160) & $\ldots^{f}$ & $40.42(11)$ & 40.43 (11) \\
\hline$J_{\min } / J_{\max }$ & $1 / 75$ & $4 / 75$ & $3 / 78$ & $3 / 78$ \\
\hline$K_{a}^{\min } / K_{a}^{\max }$ & $0 / 37$ & $0 / 37$ & $0 / 33$ & $0 / 37$ \\
\hline$N^{g}$ & 354 & 172 & 2538 & 2710 \\
\hline$\sigma_{\mathrm{fit}}{ }^{h} / \mathrm{MHz}$ & 0.081 & 0.065 & 0.019 & 0.028 \\
\hline
\end{tabular}

Notes. ${ }^{(a)}$ Whole data set from Marstokk et al. (2000). ${ }^{(b)}$ Data from Marstokk et al. (2000) when the misassigned lines and hyperfine-split lines were removed. ${ }^{(c)}$ Data solely from the present work. ${ }^{(d)}$ A global fit including the data from the present work and those from the second column of this table. ${ }^{(e)}$ The numbers in parentheses are the parameter uncertainties in units of the last decimal digit. SPFIT/SPCAT program package (Pickett 1991) was used for the analysis. ${ }^{(f)}$ Fixed to zero. ${ }^{(g)}$ Number of distinct frequency lines in the fit. ${ }^{(h)}$ Root mean square deviation of the fit.

Table A.4. List of the measured transitions of skew acrylamide.

\begin{tabular}{|c|c|c|c|c|c|c|c|c|c|c|c|c|c|}
\hline$J^{\prime}$ & $K_{a}^{\prime}$ & $K_{c}^{\prime}$ & $v^{\prime c}$ & $J^{\prime \prime}$ & $K_{a}^{\prime \prime}$ & $K_{c}^{\prime \prime}$ & $v^{\prime \prime a}$ & $v_{\mathrm{obs}}(\mathrm{MHz})^{b}$ & $v_{\text {obs }}-v_{\text {calc }}(\mathrm{MHz})^{c}$ & $u_{\text {obs }}(\mathrm{MHz})^{d}$ & $\left(v_{\text {obs }}-v_{\text {calc }}\right)_{\text {blends }}(\mathrm{MHz})^{e}$ & Weight $t^{f}$ & Notes $^{g}$ \\
\hline 8 & 5 & 3 & 0 & 7 & 5 & 2 & 0 & 59501.6900 & -0.0654 & 0.100 & & & $(1)$ \\
\hline 15 & 1 & 15 & 0 & 14 & 1 & 14 & 0 & 94032.4063 & -0.0025 & 0.050 & & & (2) \\
\hline 26 & 1 & 25 & 0 & 25 & 2 & 24 & 0 & 166718.5703 & 0.0045 & 0.030 & -0.0114 & 0.29 & (2) \\
\hline 26 & 2 & 25 & 0 & 25 & 2 & 24 & 0 & 166718.5703 & -0.0053 & 0.030 & -0.0114 & 0.21 & (2) \\
\hline 26 & 2 & 25 & 0 & 25 & 1 & 24 & 0 & 166718.5703 & -0.0273 & 0.030 & -0.0114 & 0.29 & (2) \\
\hline 26 & 1 & 25 & 0 & 25 & 1 & 24 & 0 & 166718.5703 & -0.0174 & 0.030 & -0.0114 & 0.21 & (2) \\
\hline 16 & 0 & 16 & 1 & 15 & 1 & 15 & 1 & 100553.9229 & 0.0256 & 0.050 & & & (2) \\
\hline 26 & 7 & 20 & 1 & 25 & 7 & 19 & 1 & 196306.1152 & 0.0069 & 0.030 & & & (2) \\
\hline 22 & 17 & 5 & 1 & 21 & 16 & 6 & 1 & 370327.0981 & -0.0034 & 0.030 & -0.0034 & 0.50 & (2) \\
\hline 22 & 17 & 6 & 1 & 21 & 16 & 5 & 1 & 370327.0981 & -0.0034 & 0.030 & -0.0034 & 0.50 & (2) \\
\hline
\end{tabular}

Notes. ${ }^{(a)} v=0$ corresponds to $0^{+}$state and $v=1$ to $0^{-}$state. ${ }^{(b)}$ Observed frequency. ${ }^{(c)}$ Observed minus calculated frequency. ${ }^{(d)}$ Uncertainty of the observed frequency. ${ }^{(e)}$ Observed minus calculated frequency for blends. ${ }^{(f)}$ Intensity weighting factor for blended transitions. ${ }^{(g)}$ Source of the data: (1) Marstokk et al. (2000), (2) This work. This table is available in its entirety at the CDS. 

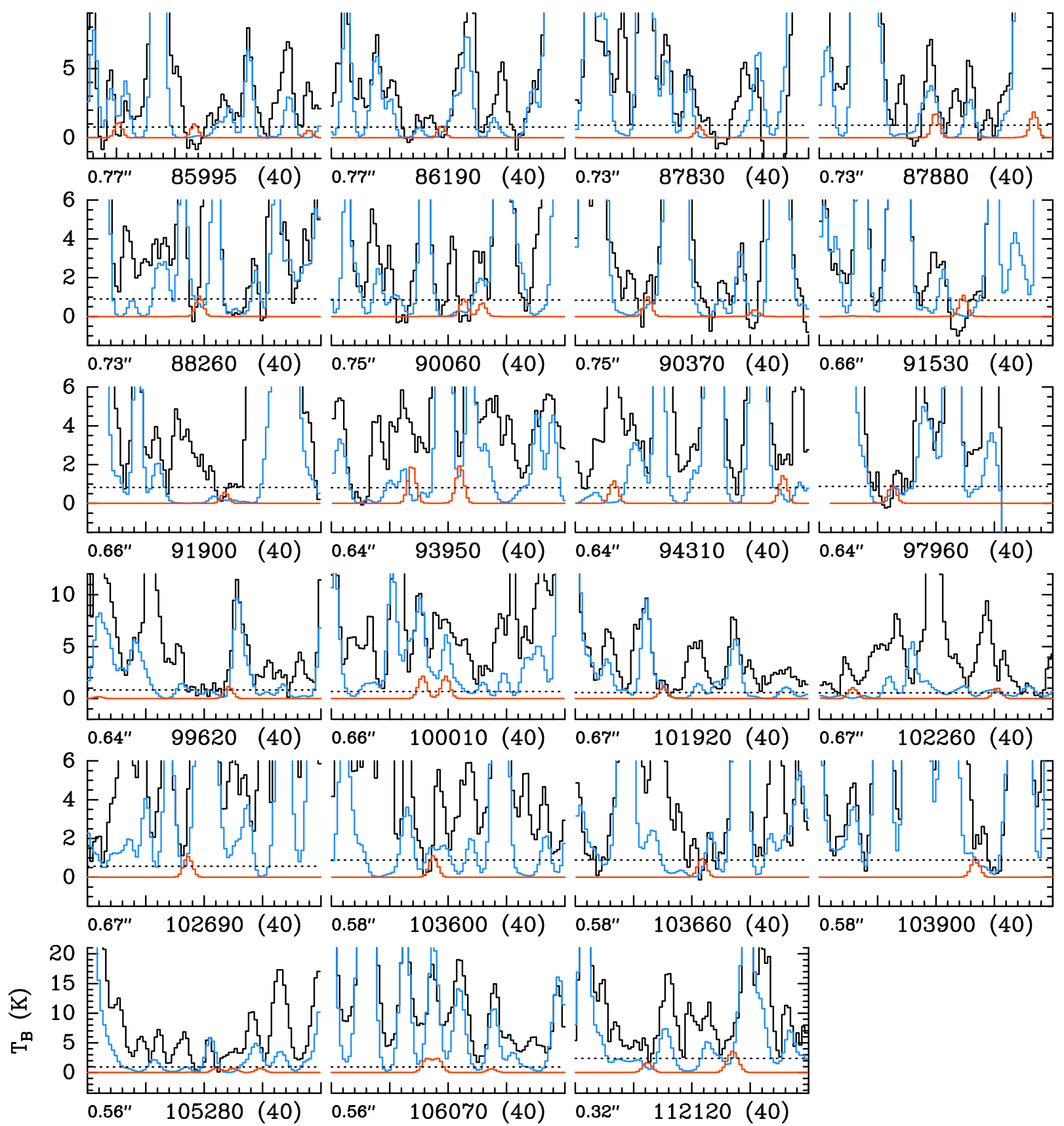

Fig. B.1. Selection of transitions of acrylamide, syn- $\mathrm{C}_{2} \mathrm{H}_{3} \mathrm{C}(\mathrm{O}) \mathrm{NH}_{2}, v=0$ covered by the ReMoCA survey. The synthetic spectrum of syn$\mathrm{C}_{2} \mathrm{H}_{3} \mathrm{C}(\mathrm{O}) \mathrm{NH}_{2}, v=0$ used to derive the upper limit to its column density is displayed in red and overlaid on the observed spectrum of Sgr B2(N1S) shown in black. The blue synthetic spectrum contains the contributions from all molecules identified in our survey so far, but not from the species shown in red. The central frequency and width are indicated in $\mathrm{MHz}$ below each panel. The angular resolution (HPBW) is also indicated. The $y$-axis is labeled in brightness temperature units $(\mathrm{K})$. The dotted line indicates the $3 \sigma$ noise level.

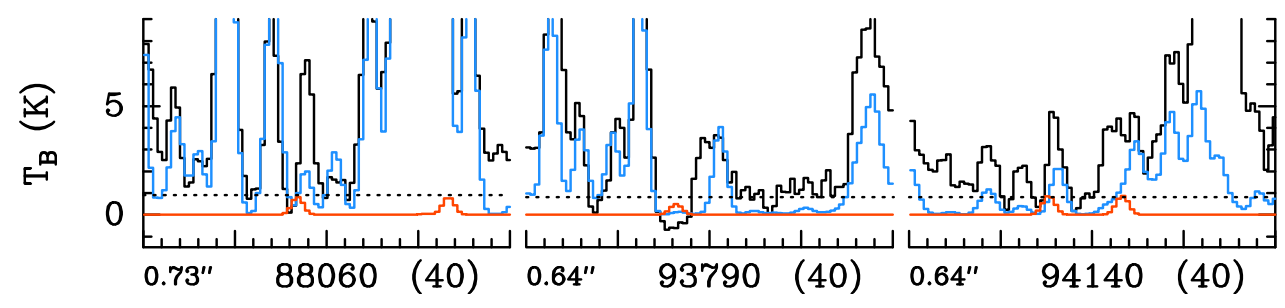

Fig. B.2. Selection of transitions of acrylamide, syn- $\mathrm{C}_{2} \mathrm{H}_{3} \mathrm{C}(\mathrm{O}) \mathrm{NH}_{2}, v_{24}=1$ toward $\mathrm{Sgr}$ B2(N1S). See the caption in Fig. B.1 for details. 

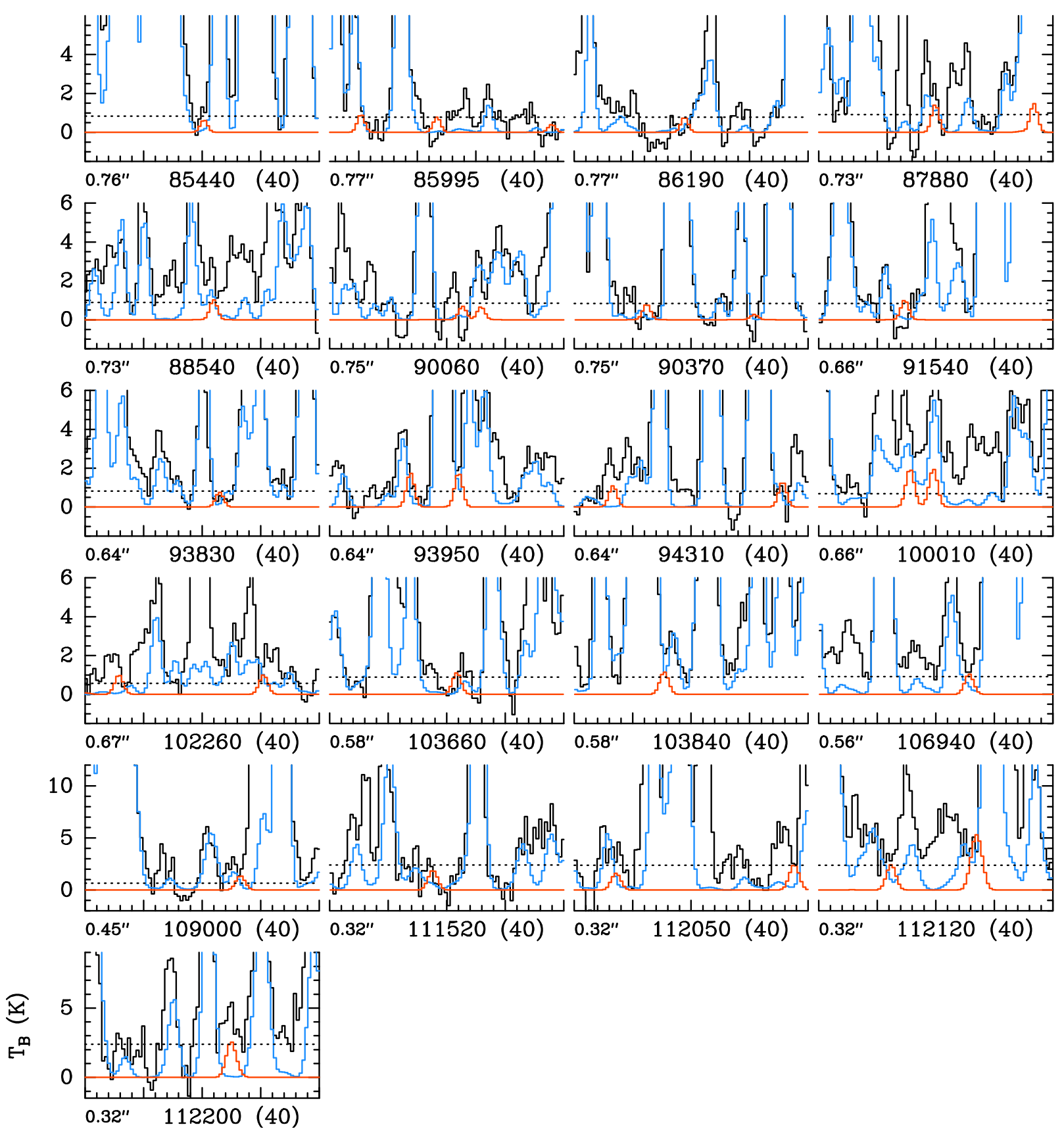

Fig. B.3. Selection of transitions of acrylamide, syn- $\mathrm{C}_{2} \mathrm{H}_{3} \mathrm{C}(\mathrm{O}) \mathrm{NH}_{2}, v=0$ toward $\mathrm{Sgr} \mathrm{B} 2(\mathrm{~N} 2)$. See the caption in Fig. B.1 for details.

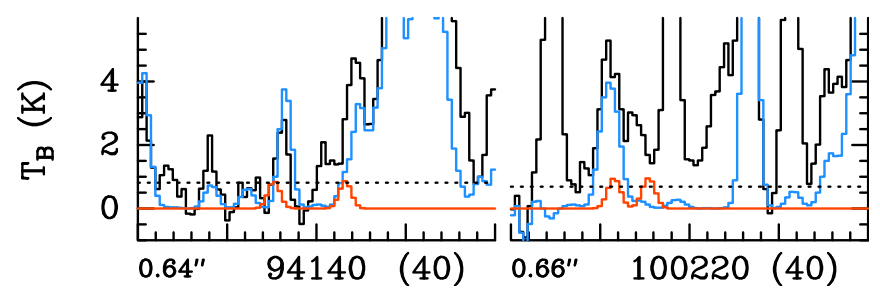

Fig. B.4. Selection of transitions of acrylamide, syn- $\mathrm{C}_{2} \mathrm{H}_{3} \mathrm{C}(\mathrm{O}) \mathrm{NH}_{2}, v_{24}=1$ toward $\mathrm{Sgr} \mathrm{B} 2(\mathrm{~N} 2)$. See the caption in Fig. B.1 for details. 

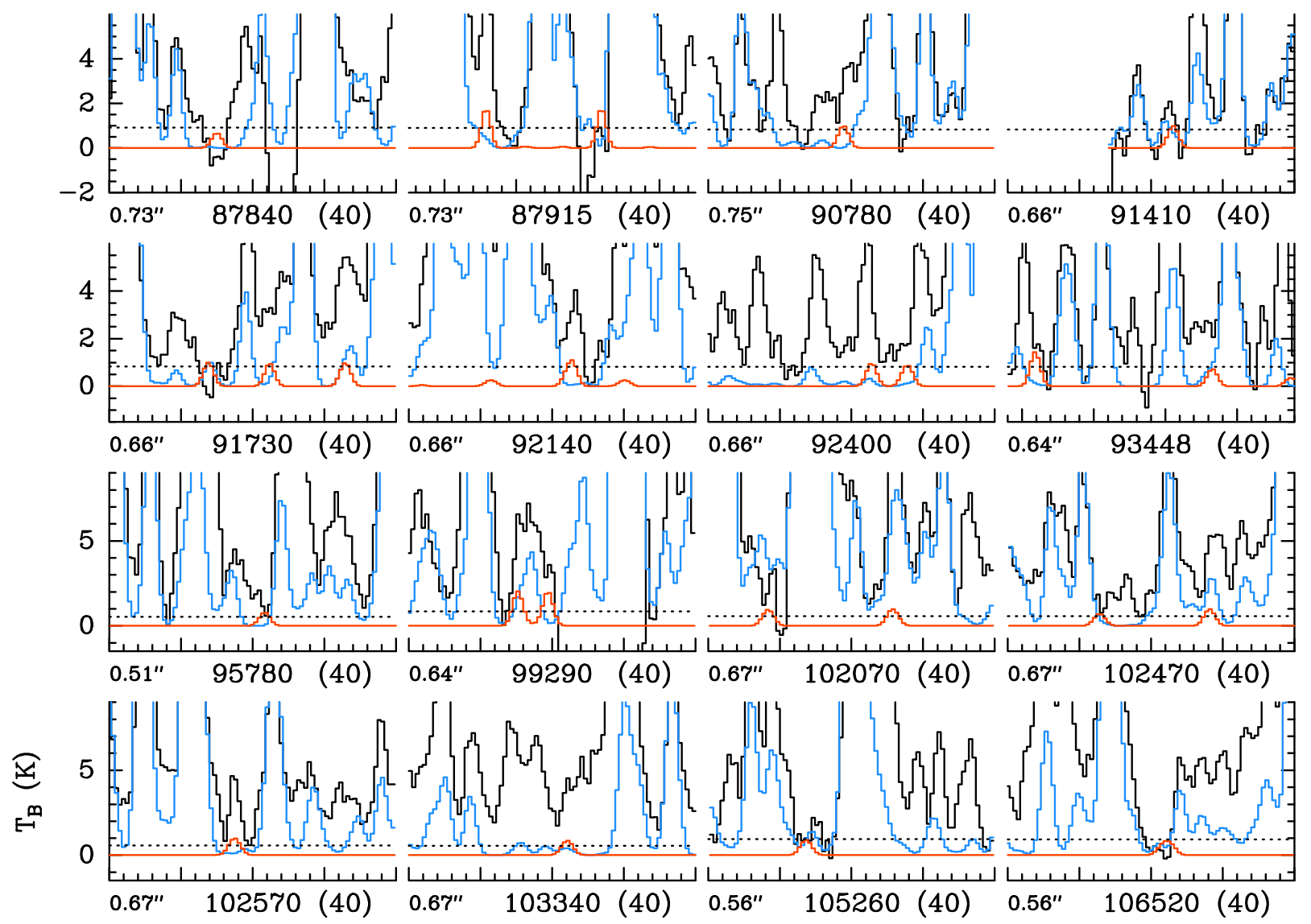

Fig. B.5. Selection of transitions of propionamide, $\mathrm{C}_{2} \mathrm{H}_{5} \mathrm{C}(\mathrm{O}) \mathrm{NH}_{2}, v=0$ toward $\mathrm{Sgr}$ B2(N1S). See the caption in Fig. B.1 for details.

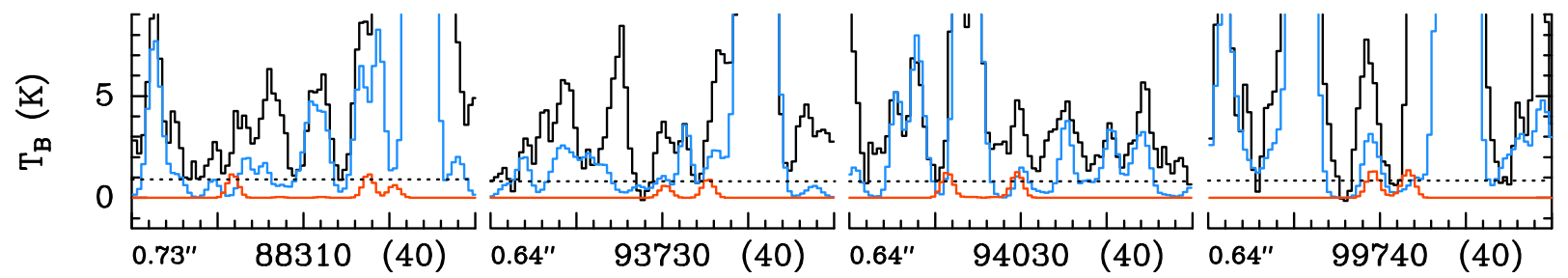

Fig. B.6. Selection of transitions of propionamide, $\mathrm{C}_{2} \mathrm{H}_{5} \mathrm{C}(\mathrm{O}) \mathrm{NH}_{2}, v_{30}=1$ toward $\mathrm{Sgr}$ B2(N1S). See the caption in Fig. B.1 for details. 

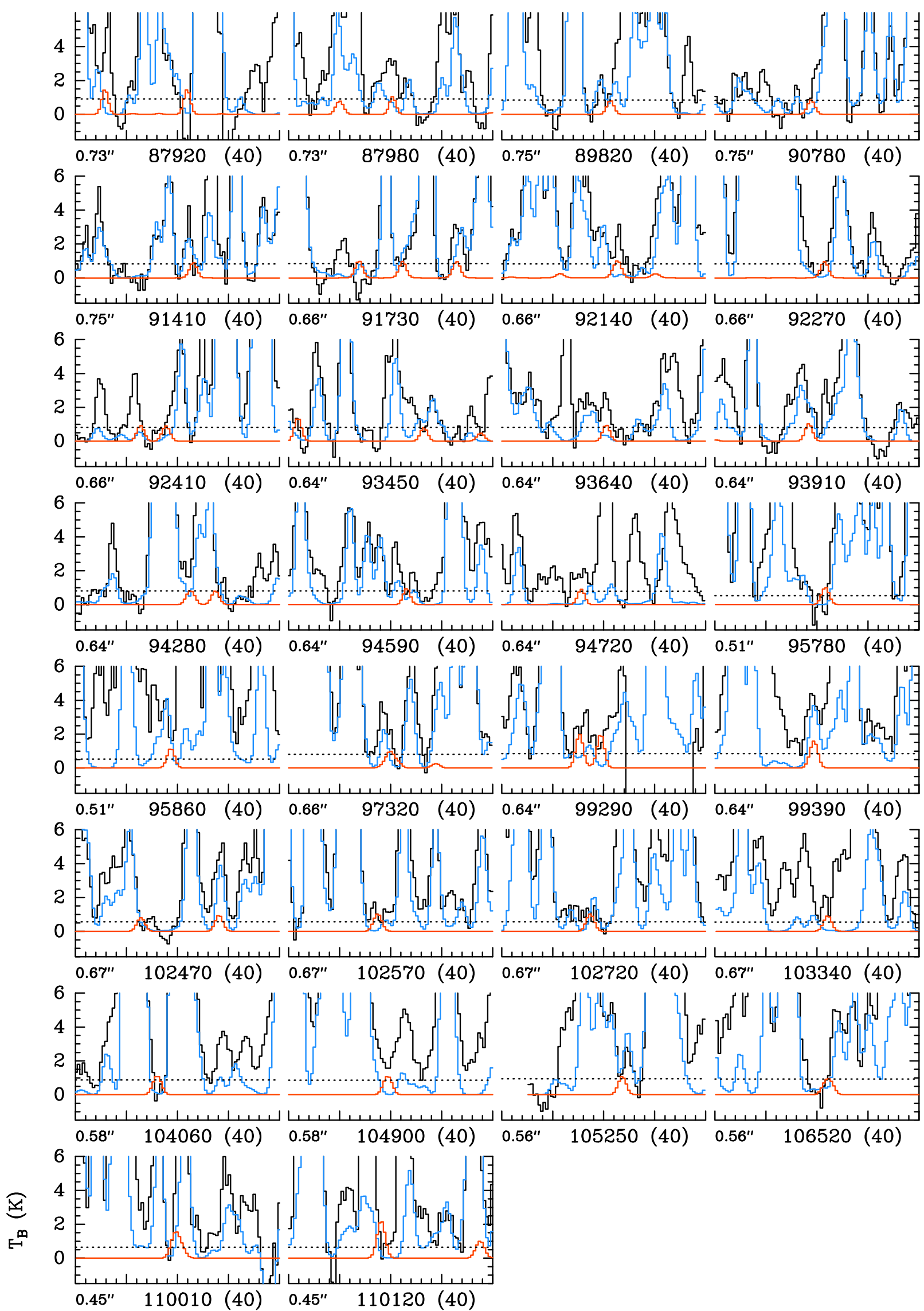

Fig. B.7. Selection of transitions of propionamide, $\mathrm{C}_{2} \mathrm{H}_{5} \mathrm{C}(\mathrm{O}) \mathrm{NH}_{2}, v=0$ toward Sgr B2(N2). See the caption in Fig. B.1 for details. 

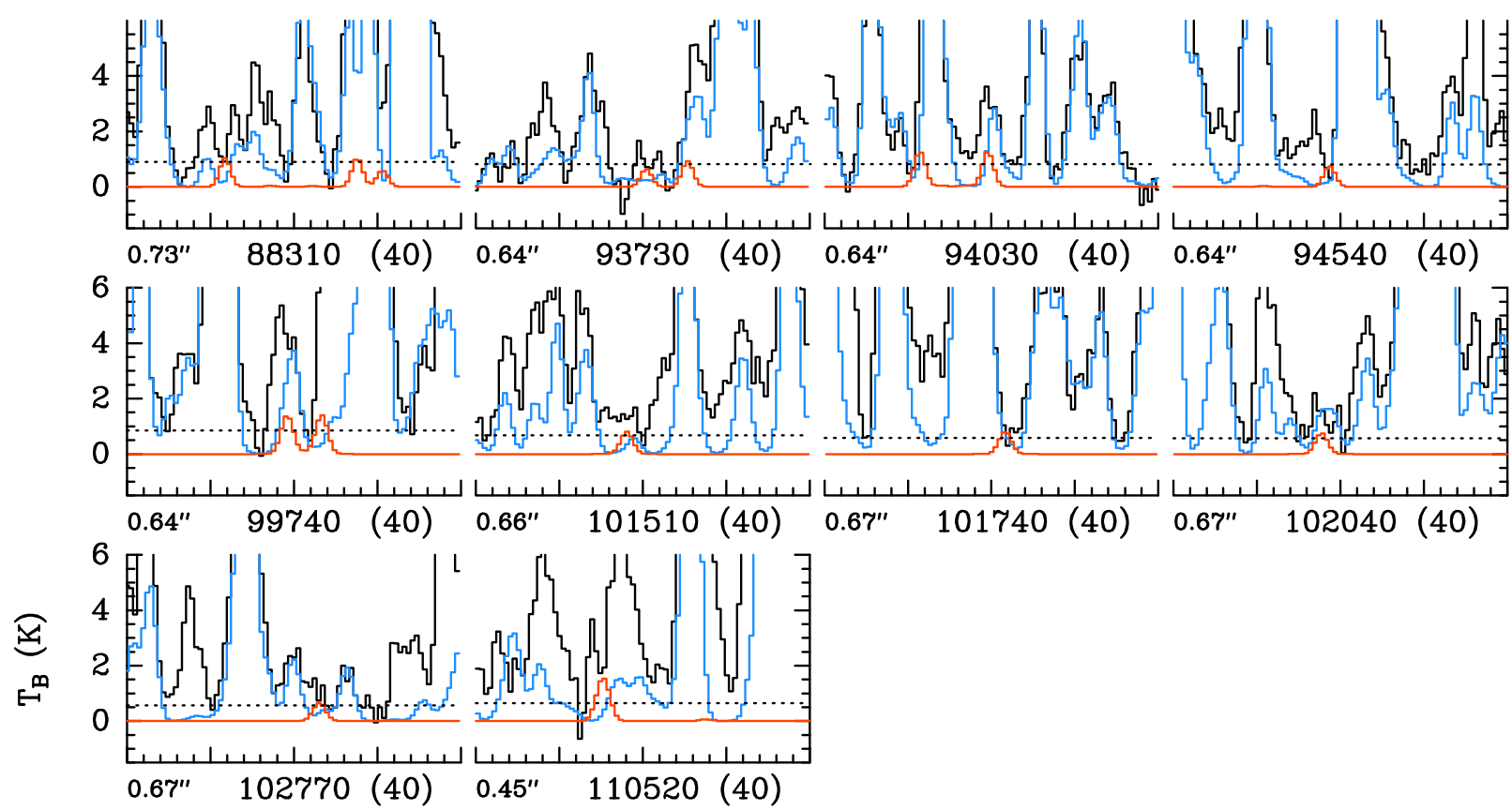

Fig. B.8. Selection of transitions of propionamide, $\mathrm{C}_{2} \mathrm{H}_{5} \mathrm{C}(\mathrm{O}) \mathrm{NH}_{2}, v_{30}=1$ toward $\mathrm{Sgr} \mathrm{B} 2(\mathrm{~N} 2)$. See the caption in Fig. B.1 for details. 

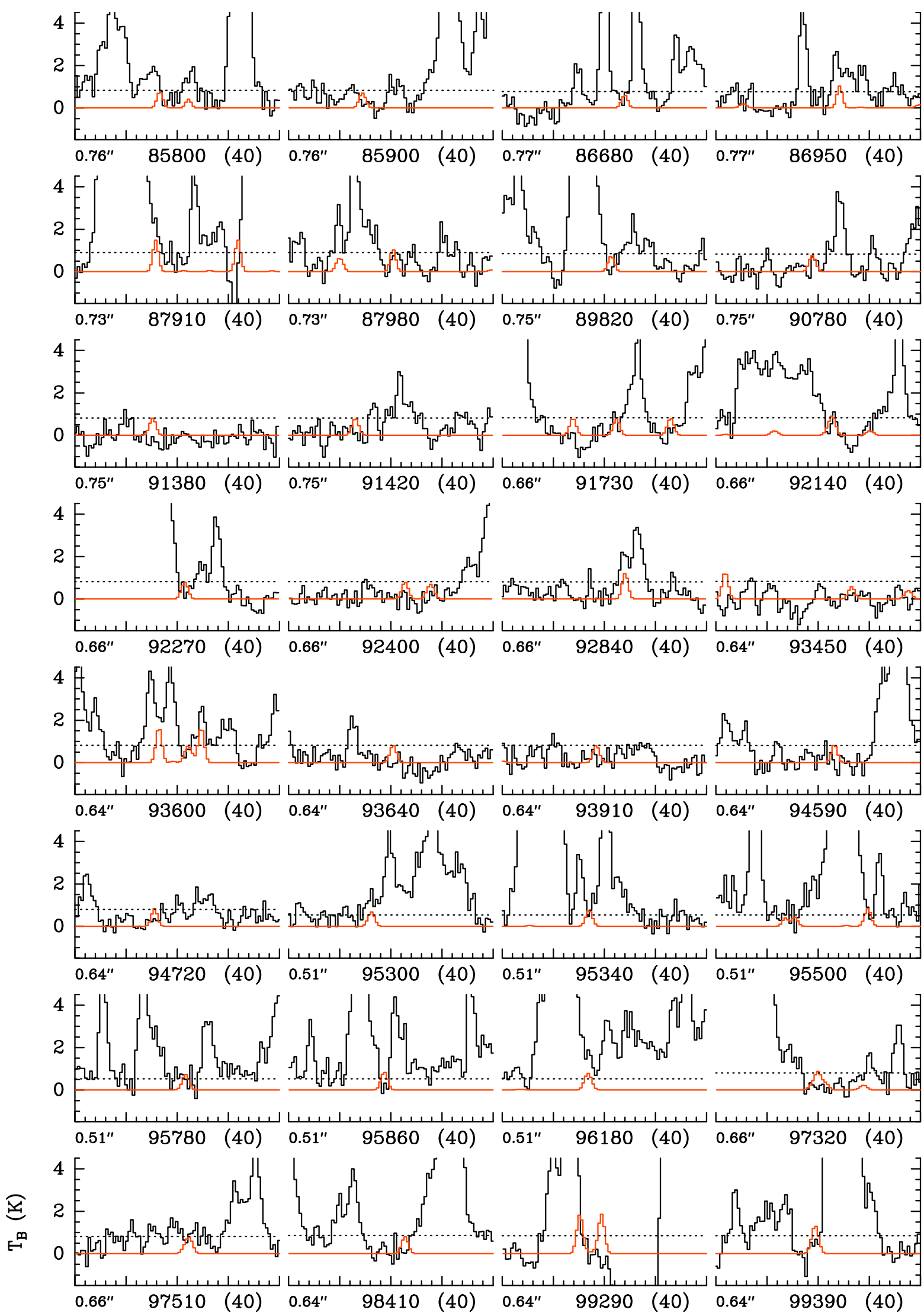

Fig. B.9. Selection of transitions of propionamide, $\mathrm{C}_{2} \mathrm{H}_{5} \mathrm{C}(\mathrm{O}) \mathrm{NH}_{2}, v=0$ covered by the ReMoCA survey. The synthetic spectrum of $\mathrm{C}_{2} \mathrm{H}_{5} \mathrm{C}(\mathrm{O}) \mathrm{NH}_{2}, v=0$ used to derive the upper limit to its column density is displayed in red and overlaid on the black spectrum observed toward the equatorial offset position $\left(1.58^{\prime \prime},-2.72^{\prime \prime}\right)$ relative to the phase center. The central frequency and width are indicated in $\mathrm{MHz}$ below each panel. The angular resolution (HPBW) is also indicated. The $y$-axis is labeled in brightness temperature units (K). The dotted line indicates the $3 \sigma$ noise level. 
L. Kolesniková et al.: The rotational spectrum and ISM search of acrylamide
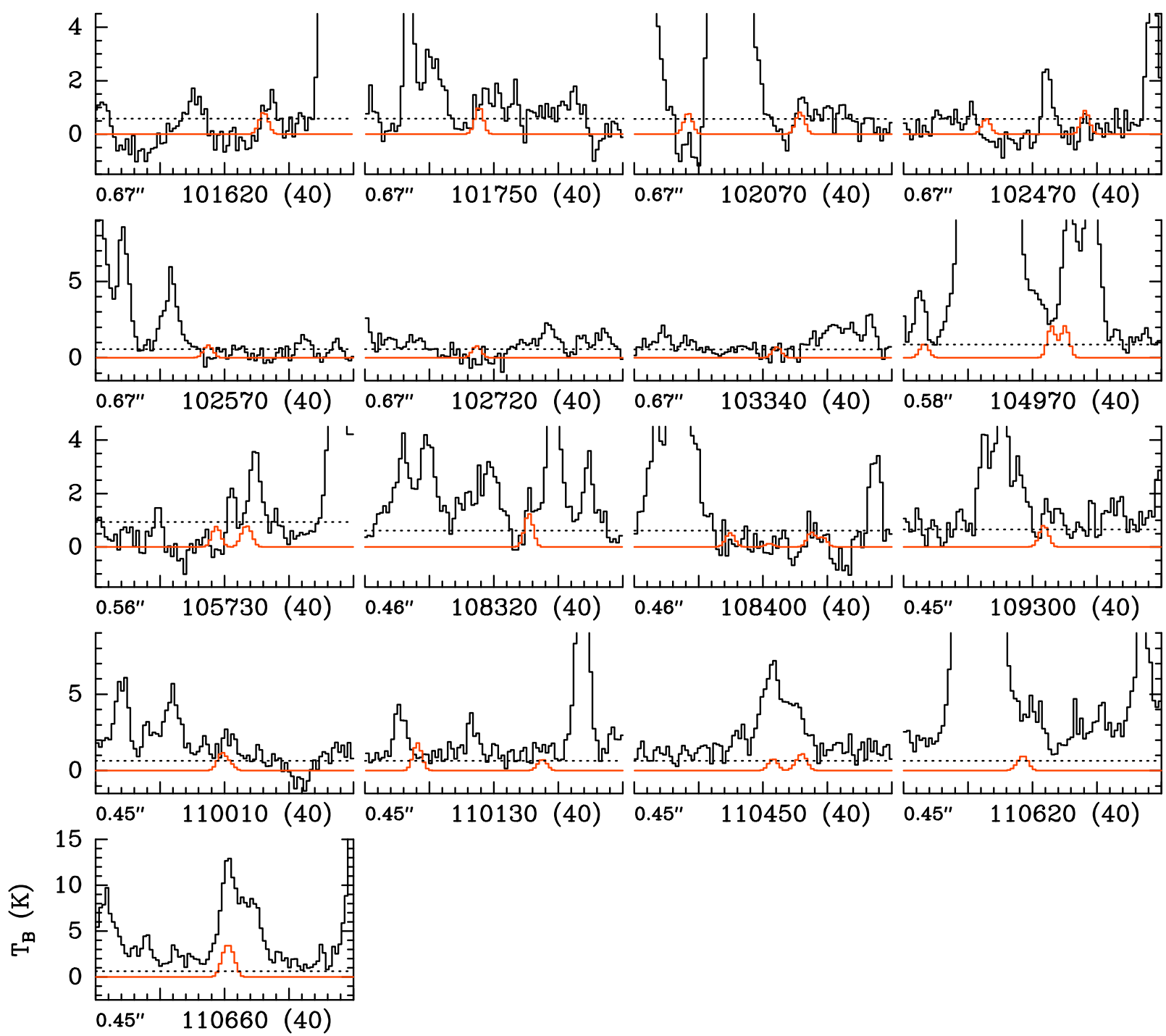

Fig. B.9. continued. 

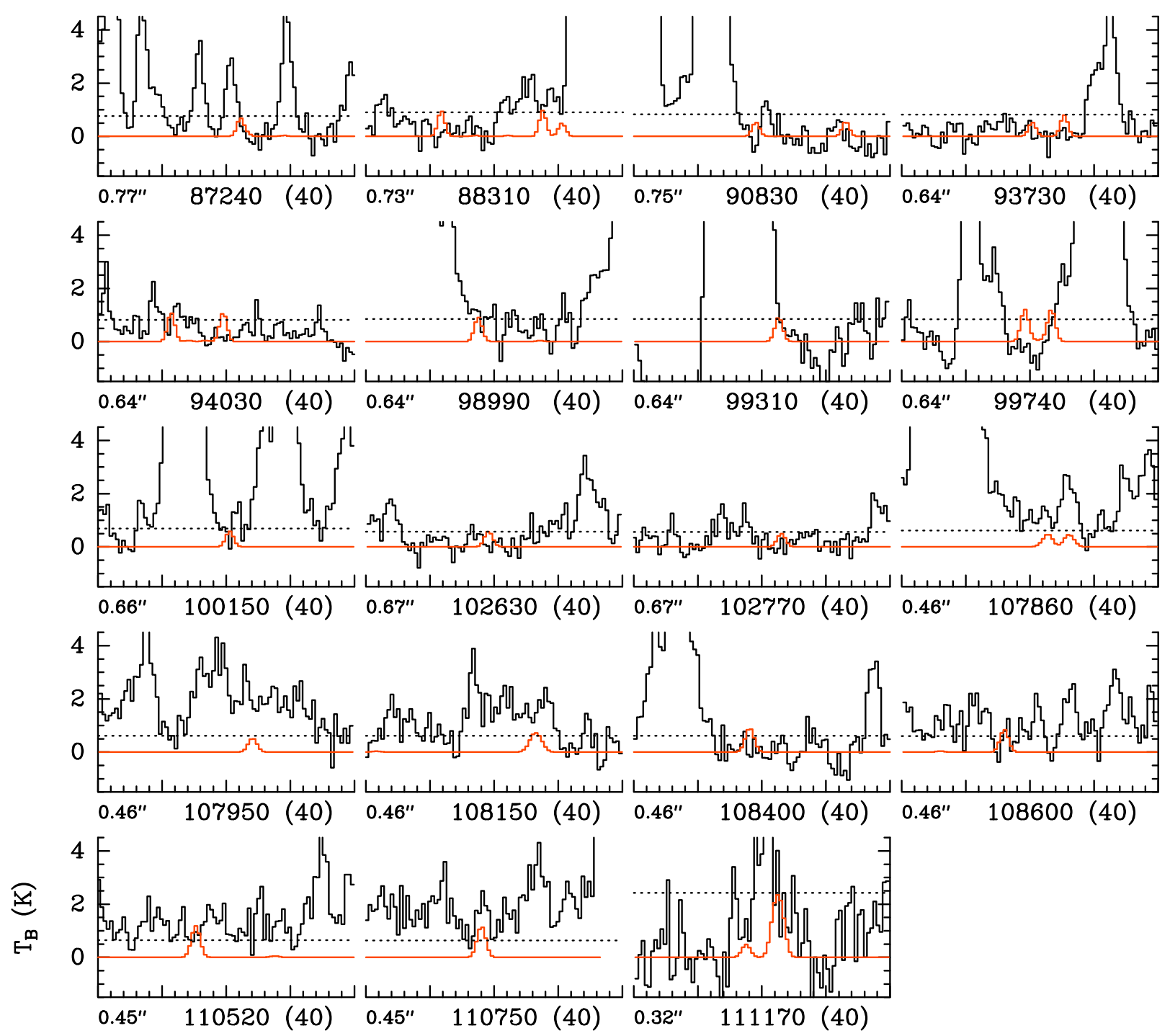

Fig. B.10. Selection of transitions of propionamide, $\mathrm{C}_{2} \mathrm{H}_{5} \mathrm{C}(\mathrm{O}) \mathrm{NH}_{2}, v_{30}=1$. See the caption in Fig. B.9 for details. 